\title{
Thermodynamics of structurally disordered $s-d$ model
}

\author{
Yu.K.Rudavskii, G.V.Ponedilok, L.A.Dorosh \\ National University "Lvivska Politechnika", \\ 12 S.Bandera Str., 79013 Lviv, Ukraine
}

Received May 5, 2004, in final form October 22, 2004

\begin{abstract}
Spin-electron exchange model is generalized and used for description of magnetic states of amorphous substitutional alloys with the structural disorder of the liquid type. A scheme of consistently accounting for the contributions of structural fluctuations to the thermodynamic functions and observable quantities is considered. Using the perturbation theory, the functional of thermodynamic potential is constructed as a functional power series. In the random phase approximation (RPA), the grand thermodynamic potential of the model is calculated. Self-consistency conditions are given, from which equations for magnetizations and critical temperature of the paramagnetic-ferromagnetic transition are obtained.
\end{abstract}

Key words: $s-d$-model, ferromagnetic alloys, free energy, magnetization, Curie temperature, functional integral

PACS: 72.15.C, 73.20.H, 75.10, 75.30.E, 82.65.Y

\section{Introduction}

The exchange $s$ - $d$ model is used for description of electric and magnetic properties of compounds containing transition and rare-earth elements. Introduced by Shubin and Vonsovsky, the exchange $s$ - $d$ model was further developed by Zener, Turov, Kasuya, Yosida and others and found an increasing number of applications in various fields of solid state physics. Initially it was proposed for description of electric and magnetic properties of transition $d$-metals [1]; today it forms a basis for a theory of magnetism in rare-earth metals and magnetic semiconductors $[2,3]$ and Kondo systems [4]. From the theoretical point of view, the importance of $s$ - $d$ model is confirmed by the fact that it predicts many beautiful and non-trivial effects: formation of spin polarons, ferrons, fluctuons in magnetic semiconductors, occurrence of spin-glass state in the dissolved metal alloys, Kondo-effect, etc. Applications of the $s$ - $d$ model are not restricted only to the listed objects. The pseudo-spin-electron model in which the role of spin operators is played by formal pseudo-spin operators, describing the generalized degrees of freedom of a many-body system, is used for 
description of a strongly correlated electron system and compounds in which the phenomenon of high-temperature superconductivity is observed $[5,6]$.

Most of papers (see [1-5,7] and references therein) concern the systems with one magnetic impurity in a metal or semiconductor, or the so-called periodic $s$ - $d$ model. These theoretical investigations of $s$ - $d$ model are mainly aimed at calculating the static and dynamic correlation functions. However, only in a few theoretical works the thermodynamics of $s$ - $d$ model is studied, and phase transitions for this model are investigated and classified. In particular, in [8] the high-temperature expansions are constructed for free energy of the simplified model, where a transeverse part of the spin-electron interaction operator was neglected. Authors of [9] have presented a critical survey of different versions of perturbation theory which were used to calculate the free energy. In [10], by using a method developed in $[11,12]$, the functional of thermodynamic potential for the generalized periodical $s$ - $d$ model was constructed.

The present work continues the investigation [13] of the structurally disordered $s$ - $d$ model. In contrast to the previous work, within the perturbation theory the contribution to the thermodynamic potential from correlational spin-electron interactions as well as the effects caused by scattering of conductivity electrons on structure fluctuations are taken into account.

\section{The spin-electron model for the amorphous binary magnet}

In the traditional classical sense, the $s$ - $d$ model of ferromagnetic crystal metals describes the interaction of conductivity electrons with localized magnetic moments of atoms by an exchange mechanism $[1,2]$. Thus states of conductivity electrons are described by plane waves or Bloch wave packages. To apply this model to structurally disordered ferromagnetic alloys, it is necessary to introduce certain specifications and generalizations which are essential for a correct description of this class of substances.

\subsection{The Hamiltonian}

Amorphous binary alloy of $N$ atoms in the $V \subset \mathbb{R}^{3}$ volume is considered. Part of these atoms have magnetic moments (hereafter - a magnetic subsystem of the alloy), but the other ones do not have any (nonmagnetic subsystem of the alloy). The atom coordinates $\left(\mathbf{R}_{1}, \ldots, \mathbf{R}_{N}\right) \equiv \mathbf{R}^{N} \in V$ can have random values. Ratio of the numbers of magnetic and nonmagnetic atoms is given by concentration $c(0 \leqslant c \leqslant 1)$.

The microscopic model of an amorphous magnet takes into account the presence of two quantum interacting subsystems - i.e., of localized atom spins and of conduction electrons. The model Hamiltonian is given by

$$
\hat{H}=\hat{H}_{\mathrm{s}}+\hat{H}_{\mathrm{el}}+\hat{H}_{\mathrm{el}-\mathrm{s}} .
$$

The first term describes the energy of the localized spin subsystem, being in the external magnetic field $h$ and in pairs interact between themselves via the Heisenberg 
exchange

$$
\hat{H}_{\mathrm{s}}=-g \mu_{\mathrm{B}} h \sum_{j=1}^{N} \hat{c}_{j} S_{j}^{z}-\frac{1}{2} \sum_{1 \leqslant i \neq j \leqslant N} J\left(\left|\mathbf{R}_{i}-\mathbf{R}_{j}\right|\right) \hat{c}_{i} \mathbf{S}_{i} \hat{c}_{j} \mathbf{S}_{j}
$$

Here $\hat{c}_{j}$ is a random variable equal to 1 or 0 if the site $j$ is occupied by a magnetic or a nonmagnetic atom, respectively; $g \mu_{\mathrm{B}}$ is the magnetic moment of atoms, and $\mathbf{S}_{j}$ is the operator of magnetic atom spin located at $\mathbf{R}_{j} \in V$. The spin operator satisfies the condition $\left|\mathbf{S}_{j}\right|^{2}=S(S+1)$, where $1 / 2 \leqslant S<\infty$ is the magnitude of the atom spin. External magnetic field $h$ is directed along the OZ axis. In terms of operators

$$
S_{\mathbf{q}}^{\alpha}=\frac{1}{\sqrt{N}} \sum_{j=1}^{N} S_{j}^{\alpha} \hat{c}_{j} \mathrm{e}^{-\mathrm{iq} \mathbf{R}_{j}}, \quad \alpha=x, y, z,
$$

the expression (2) takes the form

$$
\hat{H}_{\mathrm{s}}=\frac{1}{2} N c S(S+1) J(|\mathbf{R}|=0)-g \mu_{\mathrm{B}} h \sum_{j=1}^{N} \hat{c}_{j} S_{j}^{z}-\frac{1}{2} \sum_{\mathbf{q} \in \mathbf{\Lambda}} J_{\mathbf{q}} \mathbf{S}_{\mathbf{q}} \mathbf{S}_{-\mathbf{q}} .
$$

Fourier coefficients of the exchange spin-spin interaction of localized spins

$$
J_{\mathbf{q}}=\frac{N}{V} \int_{V} \mathrm{~d} \mathbf{r} J(|\mathbf{r}|) e^{-i \mathbf{q r}}
$$

obey the following relations: $J_{\mathbf{q}}=J_{-\mathbf{q}}$.

Electron subsystem of the alloy is described within the framework of the pseudopotential approach. The Hamiltonian of conduction electrons in our model has the form

$$
\hat{H}_{\mathrm{el}}=\sum_{\mathbf{k} \in \mathbf{\Lambda}} \sum_{\sigma= \pm 1} \mathcal{E}_{\mathbf{k}, \sigma} a_{\mathbf{k}, \sigma}^{+} a_{\mathbf{k}, \sigma}+\sum_{\mathbf{k}, \mathbf{q} \in \mathbf{\Lambda}} \sum_{\sigma \pm 1} W_{\mathbf{q}} a_{\mathbf{k}, \sigma}^{+} a_{\mathbf{k}-\mathbf{q}, \sigma}
$$

Here $a_{\mathbf{k}, \sigma}^{+},\left(a_{\mathbf{k}, \sigma}\right)$ are the creation (annihilation) Fermi operators of electrons in quantum states $\{\mathbf{k}, \sigma\}$. The wave vector $\mathbf{k}$ takes the value in quasi-continuous $\mathbf{k}$-space

$$
\Lambda=\left\{\mathbf{k}: \mathbf{k}=\sum_{1 \leqslant \alpha \leqslant 3} 2 \pi V^{-1 / 3} n_{\alpha} \mathbf{e}_{\alpha}, \quad n_{\alpha} \in \mathbb{Z}, \quad\left(\mathbf{e}_{\alpha}, \mathbf{e}_{\beta}\right)=\delta_{\alpha \beta}\right\}
$$

The quantum numbers $\sigma$ are equal to \pm 1 , corresponding to the two possible projections of electron spin on the axis of quantization $O Z$. The notation $\sigma=(\uparrow, \downarrow)$ will also be used. A spectrum of free electron gas in presence of external magnetic field $h$ is

$$
\mathcal{E}_{\mathbf{k}, \sigma}=\frac{\hbar^{2} k^{2}}{2 m}-\varkappa_{\sigma} \mu_{\mathrm{B}} h, \quad \varkappa_{\sigma}=\left\{\begin{array}{cc}
1, & \text { if } \sigma=\uparrow \\
-1, & \text { if } \sigma=\downarrow
\end{array} .\right.
$$

The matrix elements

$$
W_{\mathbf{q}}=\frac{1}{N} \sum_{j=1}^{N} \mathrm{e}^{-\mathrm{i} \mathbf{q} \mathbf{R}_{j}} w_{\mathbf{q}}, \quad w_{\mathbf{q}}=\frac{N}{V} \int_{V} \mathrm{~d} \mathbf{r} w(|\mathbf{r}|) \mathrm{e}^{-\mathrm{iq} \mathbf{r}}, \quad \mathbf{q} \in \Lambda
$$


characterize the processes of elastic scattering of electrons on the ions of the alloy; $w(|\mathbf{r}|)$ is a pseudo-potential of electron-ion interaction. For local pseudo-potentials the Fourier coefficients $w_{\mathbf{q}}$ depend only on the absolute value of the wave vector $w_{\mathbf{q}}=w_{-\mathbf{q}}$. by:

Energy of the spin-electron interaction in the coordinate representation is given

$$
\hat{H}_{\mathrm{el}-\mathrm{s}}=-\sum_{i=1}^{N_{\mathrm{e}}} \sum_{j=1}^{N} I\left(\left|\mathbf{r}_{i}-\mathbf{R}_{j}\right|\right) \mathbf{s}_{i} \hat{c}_{j} \mathbf{S}_{j}
$$

Here $\mathbf{s}_{i}$ is the operator of electron spin, localized at $\mathbf{r}_{i} \in V ; N_{\mathrm{e}}$ is the number of electrons in the system. Cartesian components of the electron spin operator are $s_{j}^{\alpha}=\hat{\sigma}^{\alpha} / 2, \alpha=x, y, z$, where $\hat{\sigma}^{\alpha}$ are the Pauli matrices. An integral of spin-electron exchange coupling in the case of contact interaction is described by expression

$$
I\left(\left|\mathbf{R}_{i}-\mathbf{r}_{j}\right|\right)=I \delta\left(\left|\mathbf{R}_{i}-\mathbf{r}_{j}\right|\right) .
$$

The interaction parameter $I$ can be positive or negative. If $I>0$, then the coupling is called ferromagnetic; if $I<0$ is antiferromagnetic. For non-contact interactions, the alternating dependence of the interaction $I\left(\left|\mathbf{R}_{i}-\mathbf{r}_{j}\right|\right)$ on the distance between electron and atom nucleus, basically, is not excluded.

The Hamiltonian of the spin-electron interaction in the representation of the second quantization by plane waves reads

$$
\hat{H}_{\mathrm{el}-\mathrm{s}}=-\frac{1}{\sqrt{N}} \sum_{\mathbf{q} \in \mathbf{\Lambda}} I_{\mathbf{q}}\left[S_{\mathbf{q}}^{z} \hat{\sigma}_{-\mathbf{q}}^{z}+\frac{1}{2}\left(S_{\mathbf{q}}^{+} \hat{\sigma}_{-\mathbf{q}}^{-}+S_{\mathbf{q}}^{-} \hat{\sigma}_{-\mathbf{q}}^{+}\right)\right] .
$$

Fourier coefficients of the spin-electron exchange interaction are

$$
I_{\mathbf{q}}=\frac{N}{V} \int_{V} I(|\mathbf{r}|) \mathrm{e}^{-\mathrm{iq \mathbf {r }}} \mathrm{d} \mathbf{r}, \quad \mathbf{q} \in \Lambda .
$$

In expression (11) the operator is

$$
S_{\mathbf{q}}^{\alpha}=\frac{1}{\sqrt{N}} \sum_{j=1}^{N} \hat{c}_{j} S_{j}^{\alpha} \mathrm{e}^{-\mathrm{iq} \mathbf{R}_{j}}, \quad \alpha=z,+,-.
$$

Here $S_{j}^{ \pm}=S_{j}^{x} \pm S_{j}^{y}$ are the components of spin flip operators. In case of lattice systems $\left(\mathbf{R}_{j} \in \mathbb{Z}^{3}\right)$ the operator (13) is the exact Fourier transform of operators $\hat{c}_{j} S_{j}^{\alpha}$. Then the wave vector $\mathbf{q}$ changes within first Brillouin zone.

In equation (11) the bilinear combinations of electron creation and annihilation Fermi operators are defined

$$
\begin{array}{ll}
\hat{\sigma}_{\mathbf{q}}^{z}=\sum_{\sigma=\uparrow, \downarrow} \frac{\varkappa_{\sigma}}{2} \hat{n}_{\mathbf{q}, \sigma}, & \hat{n}_{\mathbf{q}, \sigma}=\sum_{\mathbf{k} \in \boldsymbol{\Lambda}} a_{\mathbf{k}, \sigma}^{+} a_{\mathbf{k}+\mathbf{q}, \sigma}, \\
\hat{\sigma}_{\mathbf{q}}^{+}=\sum_{\mathbf{k} \in \boldsymbol{\Lambda}} a_{\mathbf{k}, \uparrow}^{+} a_{\mathbf{k}+\mathbf{q}, \downarrow}, & \hat{\sigma}_{\mathbf{q}}^{-}=\sum_{\mathbf{k} \in \boldsymbol{\Lambda}} a_{\mathbf{k}, \downarrow}^{+} a_{\mathbf{k}+\mathbf{q}, \uparrow} .
\end{array}
$$


Hermitian operator $\hat{\sigma}_{\mathbf{q}}^{z}$ means a Fourier component of the operator of electron spin polarization density. Fourier coefficients of the total electron density operator $\hat{n}(\mathbf{r})$ are

$$
\hat{n}_{\mathbf{q}}=\hat{n}_{\mathbf{q}, \uparrow}+\hat{n}_{\mathbf{q}, \downarrow}=\sum_{\mathbf{k} \in \mathbf{\Lambda}} \sum_{\sigma=\uparrow, \downarrow} a_{\mathbf{k}, \sigma}^{+} a_{\mathbf{k}+\mathbf{q}, \sigma}
$$

and $\widehat{N}=\lim _{|\mathbf{q}| \rightarrow \mathbf{0}} \hat{n}_{\mathbf{q}}$ is the operator of the total number of electrons.

\subsection{The structural correlation functions}

For the sake of simplicity, we shall accept that all $N$ atoms of the binary system $M_{x} N_{1-x}$ (both magnetoactive and those without localized magnetic moments) are of the same size, have identical potentials of ion-ion interaction and the same pseudo-potentials of electron-ionic interaction. We assume that magnetic exchange interactions do not effect a spatial configuration of atoms, or, at least, effect insignificantly. With such assumptions it is possible to avoid identification of the distribution of atoms of different sorts by an additional sort index. For this purpose it is sufficient to accept that the probability of the spatial allocation of atoms at points $\left\{\mathbf{R}_{1} \ldots \mathbf{R}_{N}\right\} \in V$ and the probability of random occupation of these points of space by atoms of different sorts are statistically independent. Such a model of binary system will be called an amorphous substitutional alloy.

The probability density of the distribution of atoms of the binary system in the volume $V$ will be described by the set of structural correlation functions

$$
K_{n}\left(\hat{c}_{1} \mathbf{R}_{1} ; \ldots ; \hat{c}_{n} \mathbf{R}_{n}\right)=P_{n}\left(\mathbf{R}_{1}, \ldots, \mathbf{R}_{n}\right) C_{n}\left(\frac{\hat{c}_{1} ; \ldots ; \hat{c}_{n}}{\mathbf{R}_{1} ; \ldots ; \mathbf{R}_{n}}\right), \quad n=\overline{1, N}
$$

Here $P_{n}$ is the probability density of distribution of system of atoms in space, and $C_{n}$ is the conditional probability density of distribution of different sorts of atoms over randomly chosen fixed points $\left\{\mathbf{R}_{1}, \ldots, \mathbf{R}_{N}\right\}$ of space. The magnitude of a random physical quantity averaged over all possible configurations $F\left(\hat{c}_{1} \mathbf{R}_{1} ; \ldots ; \hat{c}_{N} \mathbf{R}_{N}\right.$ ) (for instance, the free energy) is calculated over the formula

$$
\overline{F\left(\hat{c}_{1} \mathbf{R}_{1} ; \ldots ; \hat{c}_{n} \mathbf{R}_{n}\right)}=\int_{V^{n}} \sum_{\left[\hat{c}^{n}\right]} F\left(\hat{c}_{1} \mathbf{R}_{1} ; \ldots ; \hat{c}_{N} \mathbf{R}_{N}\right) K_{n}\left(\hat{c}_{1} \mathbf{R}_{1} ; \ldots ; \hat{c}_{N} \mathbf{R}_{N}\right) \mathrm{d} \mathbf{R}^{n}
$$

The symbol $\overline{(\cdots)}$ will mean hereinafter the full configurational averaging over the normalized distribution (16). Such a configurational averaging in our model can be treated as composed of two ones

$$
\overline{(\cdots)}=\left\langle\langle(\cdots)\rangle_{\mathrm{c}}\right\rangle_{\mathbf{R}}
$$

where $\langle(\cdots)\rangle_{\mathbf{R}}$ means the averaging over the distribution of the atoms in space, and $\langle(\cdots)\rangle_{\mathrm{c}}$ means the averaging over the occupation of the fixed points of space by atoms of different sorts. 
It is convenient to describe the structure of amorphous system by the correlation functions

$$
K_{n}\left(\mathbf{k}_{1} ; \ldots ; \mathbf{k}_{n}\right)=\left[\frac{1}{\sqrt{N}}\right]^{2-n} \overline{\overline{\hat{c}_{\mathbf{k}_{1}} \ldots \hat{c}_{\mathbf{k}_{n}}}},
$$

where the notation $\overline{\overline{(\cdots)}}$ stands for the irreducible average over the random configurations. The values

$$
\hat{c}_{\mathbf{k}}=\frac{1}{\sqrt{N}} \sum_{j=1}^{N} \hat{c}_{j} \mathrm{e}^{-\mathrm{ikR_{j }},} \quad \mathbf{k} \neq \mathbf{0} .
$$

with accuracy to the factor $\sqrt{N}$ are the Fourier components of fluctuations of magnetic atoms concentrations. In the case of lattice structure which corresponds to a crystal binary substitutional alloy

$$
\left\langle\hat{c}_{\mathbf{k}_{1}} \cdots \hat{c}_{\mathbf{k}_{n}}\right\rangle_{\mathrm{c}}=\left\langle\hat{c}_{j_{1}} \cdots \hat{c}_{j_{n}}\right\rangle_{\mathrm{c}} \delta_{\mathbf{k}_{1}+\cdots+\mathbf{k}_{n}, \mathbf{0}}
$$

Configurational average $\left\langle\hat{c}_{j_{1}} \ldots \hat{c}_{j_{n}}\right\rangle_{\mathrm{c}}$ over the distribution of atoms of different sorts can be expressed through the irreducible averages: $P_{m}(c)=\left\langle\hat{c}_{j_{1}} \ldots \hat{c}_{j_{m}}\right\rangle_{\mathrm{c}}^{\mathrm{irr}}$, the generating functional of which is

$$
g(t ; c)=\sum_{m \geqslant 1} P_{m}(c) \frac{t^{m}}{m !}=\ln \left(1-c+c \mathrm{e}^{t}\right) .
$$

Some first cumulants are $P_{1}(c)=c, P_{2}(c)=c(1-c), P_{3}(c)=c(1-c)(1-2 c)$, $P_{4}(c)=c(1-c)\left(1-6 c+6 c^{2}\right)$, etc.

The structural correlation functions are reduced to the form:

$$
\begin{aligned}
K_{2}\left(\mathbf{k}_{1} ; \mathbf{k}_{2}\right)= & c\left[1-c+c S_{2}\left(k_{1}\right)\right] \delta_{\mathbf{k}_{1}+\mathbf{k}_{2}, \mathbf{0}} \\
K_{3}\left(\mathbf{k}_{1} ; \mathbf{k}_{2} ; \mathbf{k}_{3}\right)= & {\left[c(1-c)(1-2 c)-c^{2}(1-c)\left\{S_{2}\left(k_{1}\right)+S_{2}\left(k_{2}\right)+S_{2}\left(k_{3}\right)\right\}\right.} \\
& \left.+c^{3} S_{3}\left(\mathbf{k}_{1} ; \mathbf{k}_{2} ; \mathbf{k}_{3}\right)\right] \delta_{\mathbf{k}_{1}+\mathbf{k}_{2}+\mathbf{k}_{3}, \mathbf{0}}, \quad \text { etc. }
\end{aligned}
$$

Correlation functions of the atomic density fluctuations defined as

$$
S_{m}\left(\mathbf{k}_{1}, \ldots, \mathbf{k}_{m}\right) \delta_{\mathbf{k}_{1}+\cdots+\mathbf{k}_{m}, \mathbf{0}} \stackrel{\text { def }}{=}\left[\frac{1}{\sqrt{N}}\right]^{2-m}\left\langle\rho_{\mathbf{k}_{1}} \ldots \rho_{\mathbf{k}_{m}}\right\rangle_{\mathbf{R}}^{\text {irr }}
$$

cannot be calculated ab initio. Therefore we will suppose that they are phenomenological quantities.

\section{The order parameters. The effective Hamiltonian}

Thermodynamic and dynamic properties of the described spin-electron model shall be considered within the framework of the concept of order parameters. In this 
case it is necessary to make some generalizations. It is caused by the fact that the order parameters can and in some cases must be defined as functionals of random structural parameters.

Let us introduce the operators of magnetization fluctuations of a subsystem of localized atomic spins from the thermodynamic average value as

$$
\mathbf{Q}_{\mathbf{k}}=\frac{1}{\sqrt{N}} \sum_{j=1}^{N} \mathrm{e}^{-\mathrm{i} \mathbf{k} \mathbf{R}_{j}}\left(\hat{c}_{j} \mathbf{S}_{j}-\hat{c}_{j} y \mathbf{e}_{z}\right) .
$$

The thermodynamic average value of the spin of $j$ th atom depends on a spatial configuration of the system, i.e. it is the functional of coordinates of all atoms $\left\langle S_{j}^{z}\right\rangle_{T}=y_{j}\left(\hat{c}_{1} \mathbf{R}_{1}, \ldots, \hat{c}_{N} \mathbf{R}_{N}\right)$. Hereinafter the symbol

$$
\langle(\cdots)\rangle_{T}=\operatorname{Sp}_{\mathrm{s}} \operatorname{Sp}_{\mathrm{e}}\left[(\cdots) \mathrm{e}^{-\beta\left(\widehat{H}-\mu \widehat{N}_{\mathrm{e}}-\Omega\right)}\right]
$$

will mean a thermodynamic average over the grand canonical Gibbs distribution with full Hamiltonian of model, $\Omega$ is the thermodynamic potential, $\beta=\left(k_{\mathrm{B}} T\right)^{-1}$ the inversion temperature in energy units. We shall use here the approximation, when in the equation (25) the thermodynamic average $y_{j}\left(\hat{c}_{1} \mathbf{R}_{1}, \ldots, \hat{c}_{N} \mathbf{R}_{N}\right)$ is replaced by its configurationally averaged value $y=\overline{y_{j}\left(\hat{c}_{1} \mathbf{R}_{1}, \ldots, \hat{c}_{N} \mathbf{R}_{N}\right)} \equiv \overline{\left\langle S_{j}^{z}\right\rangle_{T}}$.

Using the same scheme we shall define the operators

$$
\widehat{\pi}_{\mathbf{q}}^{\sigma}= \begin{cases}\widehat{\sigma}_{\mathbf{q}}^{z}-\overline{\left\langle\widehat{\sigma}_{\mathbf{q}}^{z}\right\rangle_{T}}, & \sigma=z \\ \widehat{\sigma}_{\mathbf{q}}^{ \pm}, & \sigma= \pm\end{cases}
$$

the component $\widehat{\pi}_{\mathbf{q}}^{z}$ of which describes fluctuations of electron spin polarization near the thermodynamic and configurational average value. Then

$$
\overline{\left\langle\widehat{\sigma}_{\mathbf{q}}^{z}\right\rangle}=\sum_{\sigma=\uparrow, \downarrow} \frac{\varkappa_{\sigma}}{2} \delta_{\mathbf{q}, \mathbf{0}} \sum_{\mathbf{k}} \overline{\left\langle a_{\mathbf{k} \sigma}^{+} a_{\mathbf{k} \sigma}\right\rangle_{T}}=\frac{1}{2}\left(N_{\uparrow}-N_{\downarrow}\right) \delta_{\mathbf{q}, \mathbf{0}},
$$

where $N_{\sigma}=\sum_{\mathbf{k}} \overline{\left\langle a_{\mathbf{k} \sigma}^{+} a_{\mathbf{k} \sigma}\right\rangle_{T}}$ is thermodynamic and configurational average value of the total number of conductivity electrons with a spin projection $\sigma$ on the axis of quantization. We shall define the value of electron spin polarization of the unit volume (in Bohr units) as

$$
p=\frac{1}{2 V}\left(N_{\uparrow}-N_{\downarrow}\right) .
$$

Then

$$
\overline{\left\langle\widehat{\sigma}_{\mathbf{q}}^{z}\right\rangle_{T}}=p V \delta_{\mathbf{q}, \mathbf{0}} .
$$

The equation (29) is correct under the assumption that there is only a spatially homogeneous magnetic structure of electron gas in the system, i.e. ${\overline{\left\langle\widehat{\sigma}_{\mathbf{q}}^{z}\right\rangle_{T}}}_{T}=0$ at all values $\mathbf{q} \neq \mathbf{0}$. Thus, the second order parameter is the quantity $m$, which describes 
the electron spin polarization per unit volume and is determined self-consistently from the condition of a minimum of the thermodynamic potential.

In terms of operators (25) and (26), the total Hamiltonian of the $s$ - $d$ model can be rewritten in the form

$$
\widehat{H}=C+\widehat{H}_{0}+\widehat{H}_{\text {int }} .
$$

Here the non-operator part of the Hamiltonian is

$$
C=\frac{1}{2} c N S(S+1) J(0)+y p V I_{0}+\frac{y^{2}}{2} \sum_{\mathbf{k}} J_{k} \hat{c}_{\mathbf{k}} \hat{c}_{-\mathbf{k}} .
$$

An effective one-particle Hamiltonian which describes the energy of a subsystem of free conductivity electrons and energy of a subsystem of localized spins in selfconsistent fields reads

$$
\widehat{H}_{0}=\widehat{H}_{0 E}+\widehat{H}_{0 S}=\sum_{\mathbf{k}} \sum_{\sigma} \widetilde{\mathcal{E}}_{k, \sigma} a_{\mathbf{k}, \sigma}^{+} a_{\mathbf{k}, \sigma}-\sum_{j=1}^{N} \widetilde{h}_{j} \hat{c}_{j} S_{j}^{z}
$$

Effective field which acts on the spin of the atom localized at the point $\mathbf{R}_{j}$ is

$$
\widetilde{h}_{j}=g \mu_{\mathrm{B}} h+\frac{V}{N} p I_{0}+c y J_{0}+\frac{y}{2 \sqrt{N}} \sum_{\mathbf{k} \neq \mathbf{0}} J_{\mathbf{k}}\left(c_{\mathbf{k}} \mathrm{e}^{\mathrm{i} \mathbf{k} \mathbf{R}_{j}}+c_{-\mathbf{k}} \mathrm{e}^{-\mathrm{i} \mathbf{k} \mathbf{R}_{j}}\right) .
$$

The parameter $J_{0} \equiv \lim _{|\mathbf{k}| \rightarrow 0} J_{\mathbf{k}}$ characterizes the intensity of exchange spin-spin interaction. For amorphous and crystal structure it is determined, respectively, by the relations

$$
J_{0}=\frac{N}{V} \int_{V} J(|\mathbf{R}|) \mathrm{d} \mathbf{R}, \quad J_{0}=\sum_{j=1}^{N} J\left(\left|\mathbf{R}_{j}\right|\right) .
$$

For interaction only between the nearest neighbours, we have $J_{0}=z J$, where $z$ is the number of nearest neighbours, and $J$ is the exchange integral of interactions between the nearest atoms.

In the case of an ideal one-sort magnetic crystal $\left(\widehat{c}_{j} \equiv 1, \mathbf{R}_{j} \in \mathbb{Z}^{3}\right.$ for all $\left.j=\overline{1, N}\right)$ under the condition of contact spin-electron interaction the relation (33) reduces to the expression

$$
\widetilde{h}_{j} \rightarrow \widetilde{h}=g \mu_{\mathrm{B}} h+p I+y J_{0}
$$

for the usual homogeneous self-consistent field of magnetic crystals.

Renormalized due to the effects of magnetization by a subsystem of localized atom spins, the spectrum of electron gas is as follows:

$$
\widetilde{\mathcal{E}}_{k, \sigma}=\frac{\hbar^{2} k^{2}}{2 m}-\varkappa_{\sigma}\left(\mu_{\mathrm{B}} h+c y I_{0}\right)+w_{0}
$$

The constant

$$
w_{0}=\frac{N}{V} \int_{V} w(|\mathbf{r}|) \mathrm{d} \mathbf{r}
$$


is the homogeneous effective potential caused by non-coloumbic character of the pseudo-potential and does not disappear due to neutrality condition as it takes place in the model of point ions. From the condition of thermodynamic balance, the pseudo-potential should satisfy an additional condition $\left|w_{0}\right|<\infty$.

The operator of correlation interaction in (30) is

$$
\begin{aligned}
\widehat{H}_{\mathrm{int}}= & \sum_{\mathbf{k}} \sum_{\mathbf{q} \neq \mathbf{0}} \sum_{\sigma=\uparrow, \downarrow} \widetilde{W}_{q, \sigma} a_{\mathbf{k}, \sigma}^{+} a_{\mathbf{k}-\mathbf{q}, \sigma}-\frac{1}{2} \sum_{\mathbf{k} \in \mathbf{\Lambda}} J_{k} \mathbf{Q}_{\mathbf{k}} \mathbf{Q}_{-\mathbf{k}} \\
& -\frac{1}{\sqrt{N}} \sum_{\mathbf{q}} I_{q}\left[Q_{\mathbf{q}}^{z} \widehat{\pi}_{-\mathbf{q}}^{z}+\frac{1}{2}\left(Q_{\mathbf{q}}^{+} \widehat{\pi}_{-\mathbf{q}}^{-}+Q_{\mathbf{q}}^{-} \widehat{\pi}_{-\mathbf{q}}^{+}\right)\right] .
\end{aligned}
$$

The operator $\widehat{H}_{\text {int }}$ includes the term which characterizes the energy of electron scattering by ions. However, now the renormalized pseudo-potential of spin-electron scattering reads

$$
\widetilde{W}_{q, \sigma}=\frac{1}{N} \sum_{j=1}^{N} \mathrm{e}^{-\mathrm{iq} \mathbf{R}_{j}}\left(w_{q}-\varkappa_{\sigma} \hat{c}_{j} y I_{q}\right),
$$

i.e. includes the term which characterizes the exchange spin-electron interaction. It is natural that such a pseudo-potential already depends on the orientation of an electron spin with respect to the axis of quantization.

\section{The functional representation for the thermodynamic potential}

In order to go beyond the limits of the self-consistent field approximation, we use the method of functional integration developed in [11,12]. Here, in contrast to [13], the fluctuation of spin-electron interaction and the effects related to scattering of conductivity electrons by fluctuations of structure will be taken into account.

For a certain given configuration of atoms $\left\{\hat{c}_{1} \mathbf{R}_{1} ; \ldots ; \hat{c}_{N} \mathbf{R}_{N}\right\} \equiv\left[\hat{c}^{N} \mathbf{R}^{N}\right]$ the grand partition function of the model is

$$
\Xi\left[\hat{c}^{N} \mathbf{R}^{N}\right]=\mathrm{e}^{-\beta C} \operatorname{Sp}_{S} \operatorname{Sp}_{E}\left[\mathrm{e}^{-\beta\left(\widehat{H}_{0 S}+\widehat{H}_{0 E}-\mu \widehat{N}_{\mathrm{e}}\right)} \widehat{T}_{\tau} \exp \left(-\int_{0}^{\beta} \mathrm{d} \tau \widehat{H}_{\mathrm{int}}(\tau)\right)\right]
$$

where $\widehat{N}_{\mathrm{e}}$ is the operator of the total number of conductivity electrons, $\mu$ is the chemical potential of the electron subsystem. The operator $\widehat{H}_{\text {int }}(\tau)$ is written in the interaction representation, when the dependence of operators on parameter $\tau$ is given by $\widehat{A}(\tau)=\mathrm{e}^{\left(\widehat{H}_{0}-\mu \widehat{N}_{\mathrm{e}}\right) \tau} \widehat{A} \mathrm{e}^{-\left(\widehat{H}_{0}-\mu \widehat{N}_{\mathrm{e}}\right) \tau}$. The symbol $\widehat{T}_{\tau}$ stands for the operator of chronological ordering of imaginary "times" $\tau \in[0 ; \beta]$.

It is convenient to use the frequency representation in the partition function (37), having exploited the expansion into Fourier series

$$
a_{\mathbf{k}, \sigma}(\tau)=\frac{1}{\sqrt{\beta}} \sum_{\nu} a_{\mathbf{k}, \nu, \sigma} \mathrm{e}^{-\mathrm{i} \tau \nu}, \quad a_{\mathbf{k}, \sigma}^{+}(\tau)=\frac{1}{\sqrt{\beta}} \sum_{\nu} a_{\mathbf{k}, \nu, \sigma}^{+} \mathrm{e}^{\mathrm{i} \tau \nu}
$$


for Fermi operators, and

$$
\begin{gathered}
Q_{\mathbf{k}}^{\alpha}(\tau)=\frac{1}{\sqrt{\beta}} \sum_{\omega} Q_{\mathbf{k}, \omega}^{\alpha} \mathrm{e}^{-\mathrm{i} \tau \omega}, \quad \widehat{\sigma}_{\mathbf{k}}^{\alpha}(\tau)=\frac{1}{\sqrt{\beta}} \sum_{\omega} \hat{\sigma}_{\mathbf{k}, \omega}^{\alpha} \mathrm{e}^{-\mathrm{i} \tau \omega}, \\
Q_{\mathbf{k}, \omega}^{\alpha}=\frac{1}{\sqrt{\beta}} \int_{0}^{\beta} Q_{\mathbf{k}}^{\alpha}(\tau) \mathrm{e}^{\mathrm{i} \tau \omega}, \quad \widehat{\sigma}_{\mathbf{k}, \omega}^{\alpha}=\frac{1}{\sqrt{\beta}} \int_{0}^{\beta} \widehat{\sigma}_{\mathbf{k}}^{\alpha}(\tau) \mathrm{e}^{\mathrm{i} \tau \omega}, \quad \alpha=x, y, z(+,-, z) .
\end{gathered}
$$

for direct and inverse expansions into Fourier series of spin operators. In expansions (38)-(39) the Fermi frequencies have discrete values $\nu=(1+2 n) \pi / \beta, n \in \mathbb{Z}$, and Bose frequencies, accordingly, have the values $\omega=2 \pi n / \beta, n \in \mathbb{Z}$.

The Fourier components of the operators, being bilinear combinations of Fermi operators, read

$$
\begin{aligned}
\widehat{n}_{\mathbf{k}, \omega, \sigma} & =\frac{1}{\sqrt{\beta}} \sum_{\mathbf{q} \in \boldsymbol{\Lambda}} \sum_{\nu} a_{\mathbf{q}, \nu, \sigma}^{+} a_{\mathbf{q}+\mathbf{k}, \nu+\omega, \sigma}, \quad \widehat{\sigma}_{\mathbf{k}, \omega}^{z}=\frac{1}{\sqrt{2}}\left[\widehat{n}_{\mathbf{k}, \omega, \uparrow}-\widehat{n}_{\mathbf{k}, \omega, \downarrow}\right] \\
\widehat{\sigma}_{\mathbf{k}, \omega}^{+} & =\frac{1}{\sqrt{\beta N}} \sum_{\mathbf{q} \in \boldsymbol{\Lambda}} \sum_{\nu} a_{\mathbf{q}, \nu, \uparrow}^{+} a_{\mathbf{q}+\mathbf{k}, \nu+\omega, \downarrow}, \quad \widehat{\sigma}_{\mathbf{k}, \omega}^{-}=\frac{1}{\sqrt{\beta N}} \sum_{\mathbf{q} \in \boldsymbol{\Lambda}} \sum_{\nu} a_{\mathbf{q}, \nu, \downarrow}^{+} a_{\mathbf{q}+\mathbf{k}, \nu+\omega, \uparrow} .
\end{aligned}
$$

We shall use the reduced notation $\mathrm{x}=(\mathbf{k}, \omega)$ for vector four-dimensional quasicontinuous momentum-frequency space.

The contribution of an electron subsystem to the grand partition function will be calculated within the framework of the conventional thermodynamic perturbation theory [15]. Integration over the states of electron subsystem in (37) results in the following form of the grand partition function

$$
\Xi\left[\hat{c}^{N} \mathbf{R}^{N}\right]=\mathrm{e}^{-\beta\left(C+\Omega_{0 E}+\Delta \Omega_{1}\right)} \operatorname{Sp}_{S}\left[\mathrm{e}^{-\beta \widehat{H}_{0 S}} \widehat{T}_{\tau} \exp \left(-\int_{0}^{\beta} \mathrm{d} \tau \widehat{\mathcal{H}}_{\mathrm{s}-\mathrm{s}}(\tau)\right)\right]
$$

Here

$$
\Omega_{0 E}=-\frac{1}{\beta} \sum_{\mathbf{k}} \sum_{\sigma=\uparrow, \downarrow} \ln \left[1+\mathrm{e}^{-\beta\left(\widetilde{\mathcal{E}}_{k, \sigma}-\mu\right)}\right]
$$

is the thermodynamic potential of a subsystem of non-interacting electrons in the external self-consistent magnetic field. The value

$$
\Delta \Omega_{1}=\sum_{m \geqslant 2} \frac{(-1)^{m} \beta^{\frac{m}{2}-1}}{m !} \sum_{\mathrm{x}_{1}, \sigma_{1}} \cdots \sum_{\mathrm{x}_{1}, \sigma_{m}} \mathfrak{m}_{m}^{\sigma_{1} \ldots \sigma_{m}}\left(\mathrm{x}_{1} ; \ldots ; \mathrm{x}_{m}\right) \prod_{i=1}^{m} \delta_{\omega_{i}, 0} \widetilde{W}_{\mathbf{q}_{i}, \sigma_{i}}
$$

means the effective many-particle energy of interionic interaction for the given random configuration of atoms of the model. Coefficient functions

$$
\mathfrak{m}_{m}^{\sigma_{1} \ldots \sigma_{m}}\left(\mathrm{x}_{1} ; \ldots ; \mathrm{x}_{m}\right)=\left\langle\widehat{T}_{\tau} \widehat{n}_{-\mathrm{x}_{1}, \sigma_{1}} \cdots \widehat{n}_{-\mathrm{x}_{m}, \sigma_{m}}\right\rangle_{0 E}^{\mathrm{irr}}
$$

are the irreducible averages over the Gibbs' distribution with the operator $\widehat{H}_{0 E}$, constructed on operators (40). In the absence of magnetic field, in the theory of 
metals there arise the irreducible averages $\mu_{m}\left(\mathbf{k}_{1} ; \ldots ; \mathbf{k}_{m}\right)=\left\langle\widehat{T}_{\tau} \widehat{n}_{-\mathbf{k}_{1}} \cdots \widehat{n}_{-\mathbf{k}_{m}}\right\rangle_{0 E}^{\mathrm{irr}}$, constructed on operators $\widehat{n}_{\mathbf{k}}=\sum_{\sigma} \widehat{n}_{\mathbf{k}, \sigma}$, the so-called many-tail averages [15] and dynamic irreducible averages $\widetilde{\mu}_{m}\left(\mathrm{x}_{1} ; \ldots ; \mathrm{x}_{m}\right)=\left\langle\widehat{T}_{\tau} \widehat{n}_{-\mathrm{x}_{1}} \cdots \widehat{n}_{-\mathrm{x}_{m}}\right\rangle_{0 E}^{\mathrm{irr}}$, constructed on operators $\widehat{n}_{\mathrm{x}}=\sum_{\sigma} \widehat{n}_{\mathbf{k} \omega, \sigma}[16]$.

In the partition function (41) the operator $\widehat{\mathcal{H}}_{\mathrm{s}-\mathrm{s}}(\tau)$ describes the energy of spinspin interaction of a subsystem of the localized magnetic moments which includes the pair direct and effective many-particle interaction. The latter arises due to integration over the degrees of freedom of an electron subsystem. In the momentum-frequency representation this operator reads

$$
\begin{aligned}
\widehat{\mathcal{H}}_{\mathrm{s}-\mathrm{s}} & (\tau)=\sum_{m \geqslant 2} \widehat{\mathcal{H}}_{\mathrm{s}-\mathrm{s}}^{(m)}(\tau)=-\frac{\beta}{2} \sum_{\mathrm{x}} J_{k} \mathbf{Q}_{\mathbf{x}} \mathbf{Q}_{-\mathrm{x}} \\
& +\sum_{m \geqslant 2} \frac{\beta^{m}}{m ! N^{\frac{m}{2}}} \sum_{\mathrm{x}_{1}, \alpha_{1}} \cdots \sum_{\mathrm{x}_{m}, \alpha_{m}} \mathfrak{S}_{m}^{\alpha_{1} \ldots \alpha_{m}}\left(\mathrm{x}_{1} ; \ldots ; \mathrm{x}_{m}\right) \prod_{i=1}^{m} I_{\mathbf{k}_{i}} Q_{\mathrm{x}_{i}}^{\alpha_{i}} \\
& +\sum_{m \geqslant 2} \sum_{l=1}^{m-1} \frac{(-1)^{l} \beta^{m-l / 2}}{m ! N^{(m-l) / 2}} \Re_{m}^{n_{1} \ldots n_{l} \alpha_{l+1} \ldots \alpha_{m}}\left(\mathrm{x}_{1}, \ldots \mathrm{x}_{m}\right) \prod_{i=1}^{l} \delta_{\omega_{i}, 0} \\
& \times \sum_{\mathbf{k}_{i} \sigma_{i}} \widetilde{W}_{k_{i} \sigma} \prod_{j=l+1}^{m} \sum_{\mathbf{k}_{j}, \alpha_{j}} I_{k_{j}} Q_{\mathbf{k}_{j} \omega_{j}}^{\alpha_{j}} .
\end{aligned}
$$

The irreducible averages over the Gibbs' distribution of a subsystem of non-interacting electrons in the external self-consistent magnetic field

$$
\mathfrak{S}_{m}^{\alpha_{1} \ldots \alpha_{m}}\left(\mathrm{x}_{1} ; \ldots ; \mathrm{x}_{m}\right)=\left\langle\widehat{T}_{\tau} \widehat{\pi}_{-\mathrm{x}_{1}}^{\alpha_{1}} \cdots \widehat{\pi}_{-\mathrm{x}_{m}}^{\alpha_{m}}\right)_{0 E}^{\mathrm{irr}}
$$

and

$$
\mathfrak{R}_{m}^{n_{1} \ldots n_{l} \alpha_{l+1} \ldots \alpha_{m}}\left(\mathrm{x}_{1}, \ldots, \mathrm{x}_{m}\right)=\left\langle\widehat{T}_{\tau} \widehat{n}_{-\mathrm{x}_{1}, \sigma_{1}} \cdots \widehat{n}_{-\mathrm{x}_{l}, \sigma_{l}} \widehat{\pi}_{-\mathrm{x}_{l+1}}^{\alpha_{l+1}} \ldots \widehat{\pi}_{-\mathrm{x}_{m}}^{\alpha_{m}}\right\rangle_{0 E}^{\mathrm{irr}}
$$

are easily calculated with the help of the Wick theorem.

Using expression (44) one can write down the operator of the energy of pair spin-spin interaction in the momentum-frequency representation

$$
\widehat{\mathcal{H}}_{\mathrm{s}-\mathrm{s}}^{(2)}(\tau)=-\frac{\beta}{2} \sum_{\mathrm{x}}\left[\left\{J_{k}+\frac{\beta^{2}}{2} I_{k}^{2} \chi^{z z}(\mathbf{k}, \omega)\right\} Q_{\mathrm{x}}^{z} Q_{-\mathrm{x}}^{z}+\beta^{2} I_{k}^{2} \chi^{+-}(\mathbf{k}, \omega) Q_{\mathrm{x}}^{+} Q_{-\mathrm{x}}^{-}\right] .
$$

In this formula

$$
\begin{gathered}
\chi^{\sigma_{1} \sigma_{2}}(\mathbf{q}, \omega)=\frac{1}{\beta N} \sum_{\mathbf{k}, \nu} G_{0}^{\sigma_{1}}(\mathbf{k}, \nu) G_{0}^{\sigma_{2}}(\mathbf{k}-\mathbf{q}, \nu+\omega), \quad \sigma_{i}=\uparrow, \downarrow, \\
\chi^{z z}(\mathbf{q}, \omega)=\chi^{\uparrow \uparrow}(\mathbf{q}, \omega)+\chi^{\downarrow \downarrow}(\mathbf{q}, \omega)
\end{gathered}
$$

is a dynamic spin susceptibility of an electron subsystem. Here $G_{0}^{\sigma}(\mathbf{k}, \omega)=(\mathrm{i} \omega-$ $\left.\widetilde{\mathcal{E}}_{\mathbf{k}, \sigma}\right)^{-1}$ is a Fourier component of the zeroth one-electron Green function

$$
G_{0}^{\sigma}(\mathbf{k}, \tau)=-\left\langle\widehat{T}_{\tau} a_{\mathbf{k}, \sigma}(\tau) a_{\mathbf{k}, \sigma}^{+}(\tau)\right\rangle_{0 E}=-\mathrm{e}^{-\tau \widetilde{\mathcal{E}}_{\mathbf{k}, \sigma}}\left\{\begin{array}{ll}
1-n_{\mathbf{k}, \sigma}, & \tau>0 \\
-n_{\mathbf{k}, \sigma}, & \tau<0
\end{array},\right.
$$


and $n_{\mathbf{q}, \sigma}=\left[1+\mathrm{e}^{-\beta\left(\widetilde{\mathcal{E}}_{\mathbf{q}, \sigma}-\mu\right)}\right]^{-1}$ is the function of Fermi-Dirac distribution introduced above.

After summation over frequency, the susceptibility becomes

$$
\chi^{\sigma_{1} \sigma_{2}}(\mathbf{q}, \omega)=\frac{1}{N} \sum_{\mathbf{k}} \frac{n_{\mathbf{k}, \sigma_{1}}-n_{\mathbf{k}+\mathbf{q}, \sigma_{2}}}{\omega+\widetilde{\mathcal{E}}_{\mathbf{k}+\mathbf{q}, \sigma_{1}}-\widetilde{\mathcal{E}}_{\mathbf{k}, \sigma_{2}}} .
$$

Hereafter we shall take into account only the pair effective interaction and neglect the multispin interactions. Using a method developed in $[11,12]$, the grand partition function of the model will be written as a functional integral

$$
\Xi\left[\hat{c}^{N} \mathbf{R}^{N}\right]=\mathrm{e}^{-\beta\left(\Omega_{0}+\Delta \Omega_{1}\right)} \int(\mathcal{D} \varphi) \exp \left(-\frac{1}{2} \sum_{\mathrm{x}}\left|\vec{\varphi}_{\mathrm{x}}\right|^{2}-\beta \Psi[\varphi]\right)
$$

here

$$
\Omega_{0} \equiv \Omega_{0}\left[\hat{c}^{N} \mathbf{R}^{N}\right]=C-\frac{1}{\beta} \sum_{\mathbf{k}} \sum_{\sigma=\uparrow, \downarrow} \ln \left[1+\mathrm{e}^{-\beta\left(\widetilde{\mathcal{E}}_{k, \sigma}-\mu\right)}\right]-\frac{1}{\beta} \sum_{j=1}^{N} \hat{c}_{j} \ln \left[\frac{\sinh \left(\beta \widetilde{h}_{j} \frac{2 S+1}{2}\right)}{\sinh \left(\beta \widetilde{h}_{j} \frac{1}{2}\right)}\right] .
$$

A symbol of functional integration stands for

$$
\int(\mathcal{D} \varphi) \equiv \prod_{\alpha=x, y, z} \prod_{\mathrm{x}>0} \int_{-\infty}^{\infty} \frac{\mathrm{d} \varphi_{0}^{\alpha}}{\sqrt{2 \pi}} \iint_{-\infty}^{\infty} \frac{\mathrm{d} \operatorname{Re} \varphi_{\mathrm{x}}^{\alpha}}{\sqrt{\pi}} \frac{\mathrm{d} \operatorname{Im} \varphi_{\mathrm{x}}^{\alpha}}{\sqrt{\pi}}
$$

and components of field functions are

$$
\varphi_{\mathbf{q}, \omega}^{\alpha}=\frac{1}{\sqrt{\beta}} \int_{0}^{\beta} \mathrm{d} \tau \varphi_{\mathbf{q}}^{\alpha}(\tau) \mathrm{e}^{-\mathrm{i} \tau \omega}, \quad \alpha=x, y, z
$$

The functional

$$
\Psi[\varphi]=-\sum_{m \geqslant 1} \frac{\beta^{m-1}}{m !} \sum_{\mathrm{x}_{1}, \alpha_{1}} \cdots \sum_{\mathrm{x}_{m}, \alpha_{m}} \mathfrak{M}_{\alpha_{1}, \ldots, \alpha_{m}}\left(\mathrm{x}_{1} ; \ldots ; \mathrm{x}_{m}\right) \prod_{i=1}^{m} \sqrt{J_{\mathrm{x}_{i}}^{\alpha_{i}}} \varphi_{\mathrm{x}_{i}}^{\alpha_{i}} .
$$

Here the parameters of interaction are

$$
J_{\mathrm{x}}^{\alpha}=J_{k}+\frac{\beta^{2}}{2} I_{k}^{2} \chi^{\alpha,-\alpha}(\mathbf{k}, \omega)
$$

and the kernels of the functional

$$
\mathfrak{M}_{\alpha_{1} ; \ldots ; \alpha_{m}}\left(\mathrm{x}_{1} ; \ldots ; \mathrm{x}_{m}\right)=\left\langle\widehat{T}_{\tau} \widehat{Q}_{\mathrm{x}_{1}}^{\alpha_{1}} \cdots \widehat{Q}_{\mathrm{x}_{m}}^{\alpha_{m}}\right\rangle_{0 s}^{\mathrm{irr}}
$$


are the irreducible averages over the Gibbs' distribution with Hamiltonian $\widehat{H}_{0 s}$, constructed on spin operators. A few first coefficient functions read [12]:

$$
\begin{aligned}
& \mathfrak{M}_{z}\left(\mathrm{x} \mid \hat{c}^{N} \mathbf{R}^{N}\right)=M_{\mathbf{k}}=\delta_{\omega, 0} \frac{1}{\sqrt{N}} \sum_{j=1}^{N} \hat{c}_{j} \mathrm{e}^{-\mathrm{i} \mathbf{k} \mathbf{R}_{j}}\left(M_{1}\left(y_{j}\right)-y\right), \\
& \mathfrak{M}_{z z}\left(\mathrm{x}_{1}, \mathrm{x}_{2} \mid \hat{c}^{N} \mathbf{R}^{N}\right)=\delta_{\omega_{1}, 0} \delta_{\omega_{2}, 0} \frac{1}{N}\left\{\sum_{j=1}^{N} \hat{c}_{j} \mathrm{e}^{-\mathrm{i}\left(\mathbf{k}_{1}-\mathbf{k}_{2}\right) \mathbf{R}_{j}} M_{2}\left(y_{j}\right)\right. \\
& \left.-\sum_{j_{1}=1}^{N} \sum_{j_{2}=1}^{N} \hat{c}_{j_{1}} \hat{c}_{j_{2}} \mathrm{e}^{-\mathrm{i} \mathbf{k}_{1} \mathbf{R}_{j_{1}}+i \mathbf{k}_{2} \mathbf{R}_{j_{2}}}\left[M_{1}\left(y_{j_{1}}\right) y+M_{1}\left(y_{j_{2}}\right) y+y^{2}\right]\right\} \\
& \mathfrak{M}_{-+}\left(\mathrm{x}_{1}, \mathrm{x}_{2} \mid \hat{c}^{N} \mathbf{R}^{N}\right)=\delta_{\omega_{1}+\omega_{2}, 0} \frac{2}{N} \sum_{j=1}^{N} \hat{c}_{j} \mathrm{e}^{-\mathrm{i}\left(\mathbf{k}_{1}+\mathbf{k}_{2}\right) \mathbf{R}_{j}} M_{1}\left(y_{j}\right) K_{0}^{-+}\left(\omega_{2} \mid y_{j}\right) \text {. }
\end{aligned}
$$

Here the ideal spin temperature Green function is

$$
K_{0}^{-+}\left(\tau_{1}-\tau_{2} \mid y_{j}\right) \stackrel{\text { def }}{=} \frac{\left\langle\widehat{T}_{\tau} S_{j}^{-}\left(\tau_{1}\right) S_{j}^{+}\left(\tau_{2}\right)\right\rangle_{0}}{2\left\langle S_{j}^{z}\right\rangle_{0}}=\sum_{\omega} K_{0}^{-+}\left(\omega \mid y_{j}\right) \mathrm{e}^{\mathrm{i} \omega\left(\tau_{1}-\tau_{2}\right)},
$$

and its Fourier components are

$$
K_{0}^{-+}\left(\omega \mid y_{j}\right)=\left(y_{j}-\mathrm{i} \beta \omega\right)^{-1} .
$$

We consider here only the Gaussian approximation when we take into account only

$$
\begin{aligned}
\mathcal{F}_{1}[\varphi]= & \sum_{\mathrm{x}} \sqrt{J_{\mathrm{x}}^{z}} \mathfrak{M}_{z}\left(\mathrm{x} \mid \hat{C}^{N} \mathbf{R}^{N}\right) \varphi_{\mathrm{x}}^{z} \\
\mathcal{F}_{2}[\varphi]= & \sum_{\mathrm{x}_{1}} \sum_{\mathrm{x}_{2}} \sqrt{J_{\mathrm{x}_{1}}^{z} J_{\mathrm{x}_{2}}^{z}} \mathfrak{M}_{z z}\left(\mathrm{x}_{1}, \mathrm{x}_{2} \mid \hat{c}^{N} \mathbf{R}^{N}\right) \varphi_{\mathrm{x}_{1}}^{z} \varphi_{\mathrm{x}_{2}}^{z} \\
& +\frac{1}{2} \sum_{\mathrm{x}_{1}} \sum_{\mathrm{x}_{2}} \sqrt{J_{\mathrm{x}_{1}}^{-} J_{\mathrm{x}_{2}}^{+}} \mathfrak{M}_{-+}\left(\mathrm{x}_{1}, \mathrm{x}_{2} \mid \hat{c}^{N} \mathbf{R}^{N}\right) \varphi_{\mathrm{x}_{1}}^{-} \varphi_{\mathrm{x}_{2}}^{+}
\end{aligned}
$$

in the functional.

Correction to the thermodynamic potential (composed with the term $\Delta \Omega_{1}$ ) in Gaussian approximation reads

$$
\begin{aligned}
\Delta \Omega_{\mathrm{G}}\left[\hat{c}^{N} \mathbf{R}^{N}\right]=\beta \sum_{\mathbf{k} \in \boldsymbol{\Lambda}}\left\{w_{\mathbf{q}}^{2} \varrho_{\mathbf{k}} \varrho_{-\mathbf{k}}+y^{2} I_{q}^{2} c_{\mathbf{k}} c_{-\mathbf{k}}\right\} \chi^{z z}(\mathbf{q}, 0) \\
\quad+\frac{1}{2 \beta} \sum_{\mathrm{x}}\left\{\ln \left(1+2 J_{\mathrm{x}}^{z} \mathfrak{M}_{z z}^{0}\left(\mathrm{x},-\mathrm{x} \mid \hat{c}^{N} \mathbf{R}^{N}\right)\right)+\ln \left(1+2 J_{\mathrm{x}}^{-} \mathfrak{M}_{-+}\left(\mathrm{x},-\mathrm{x} \mid \hat{c}^{N} \mathbf{R}^{N}\right)\right)\right\} \\
\quad+\sum_{\mathrm{x}} \frac{J_{\mathrm{x}}^{z} \mathfrak{M}_{z}^{0}\left(\mathrm{x} \mid \hat{c}^{N} \mathbf{R}^{N}\right) \mathfrak{M}_{z}^{0}\left(-\mathrm{x} \mid \hat{c}^{N} \mathbf{R}^{N}\right)}{\left[1+2 J_{\mathrm{x}}^{z} \mathfrak{M}_{z z}^{0}\left(\mathrm{x},-\mathrm{x} \mid \hat{c}^{N} \mathbf{R}^{N}\right)\right]^{2}}
\end{aligned}
$$


After the configurational averaging we obtain an expression for the thermodynamic potential

$$
\begin{aligned}
& \Omega\left[\hat{c}^{N} \mathbf{R}^{N}\right]=\Omega_{0}+\beta \sum_{\mathbf{q} \in \mathbf{\Lambda}}\left[w_{\mathbf{q}}^{2} S_{2}(\mathbf{q})+I_{\mathbf{q}}^{2} y^{2} c\left(c-1+c S_{2}(\mathbf{q})\right)\right] \chi^{z z}(\mathbf{q}, 0) \\
& +\frac{N}{\beta} \sum_{\mathbf{q} \in \boldsymbol{\Lambda}} \frac{J_{\mathbf{q}, 0}^{z} c\left(c-1+c S_{2}(\mathbf{q})\right)\left[M_{1}\left(\beta h_{0}\right)-y\right]^{2}}{\left[1+2 c J_{\mathbf{q}, 0}^{z}\left\{\sqrt{N} M_{2}\left(\beta h_{0}\right)-y\left(c-1+c S_{2}(\mathbf{q})\right)\left(2 M_{1}\left(\beta h_{0}\right)+y\right)\right\}\right]^{2}} \\
& +\frac{1}{2 \beta} \sum_{\mathbf{q} \in \boldsymbol{\Lambda}} \ln \left(1+2 c J_{\mathbf{q}, 0}^{z}\left[\sqrt{N} M_{2}\left(\beta h_{0}\right)-\left(c-1+c S_{2}(\mathbf{q})\right) y\left(2 M_{1}\left(\beta h_{0}\right)+y\right)\right]\right) \\
& +\frac{1}{2 \beta} \sum_{\mathbf{q}, \omega} \ln \left(1+4 c \sqrt{N} J_{\mathbf{q} \omega}^{-} M_{1}\left(\beta h_{0}\right) K_{0}\left(\omega \mid \hat{c}^{N}\right)\right) .
\end{aligned}
$$

Here $\Omega_{0}$ is the thermodynamic potential of the self-consistent field approximation, set by expression (46) without the last term.

We can neglect the third term in this expression, as $\left[M_{1}\left(\beta h_{0}\right)-y\right] \sim \sum_{k}$; accordingly, in the Gaussian approximation the term with $\sum_{k} \sum_{p}$ drops out. To sum over frequency in the last term it is necessary to use an approximation of homogeneous media, i.e. to accept that the susceptibility does not depend on frequency: $J_{q, \omega}^{-} \simeq J_{q, 0}^{-}=J_{q}+\left(\beta^{2} / 2\right) I_{q}^{2} \chi^{\uparrow, \downarrow}(\mathbf{q}, 0)$. Then, using the integral representation of the natural logarithm, we get

$$
\begin{aligned}
\sum_{\omega} \ln \left(1+\frac{4 c \sqrt{N} M_{1}\left(\beta h_{0}\right) J_{q, 0}^{-}}{\beta\left(h_{0}-\mathrm{i} \omega\right)}\right) \\
=\frac{1}{2 \pi \mathrm{i}} \oint_{\Gamma} \mathrm{d} \xi \ln \left|\frac{\beta h_{0}+4 c \sqrt{N} M_{1}\left(\beta h_{0}\right) J_{q, 0}^{-}-\beta \xi}{\beta h_{0}-\beta \xi}\right| \frac{1}{1-\mathrm{e}^{-\beta \xi}} \\
=\frac{1}{2}\left[\ln \left(1-\mathrm{e}^{\beta h_{0}+4 c \sqrt{N} M_{1}\left(\beta h_{0}\right) J_{q, 0}^{-}}\right)-\ln \left(1-\mathrm{e}^{\beta h_{0}}\right)\right] .
\end{aligned}
$$

Here the contour of integration $\Gamma$ is chosen such that it encloses poles $\xi=h_{0}$ and $\xi=h_{0}+4 c \sqrt{N} M_{1}\left(\beta h_{0}\right) J_{q, 0}^{-} / \beta$ of analytically prolonged logarithmic function.

Finally, the averaged thermodynamic potential is

$$
\begin{aligned}
\Omega\left[\hat{c}^{N} \mathbf{R}^{N}\right] & =\Omega_{0}+\beta \sum_{\mathbf{q} \in \boldsymbol{\Lambda}}\left[w_{\mathbf{q}}^{2} S_{2}(\mathbf{q})+I_{\mathbf{q}}^{2} y^{2} c\left(c-1+c S_{2}(\mathbf{q})\right)\right] \chi^{z z}(\mathbf{q}, 0) \\
& +\frac{1}{2 \beta} \sum_{\mathbf{q} \in \boldsymbol{\Lambda}} \ln \left(1+2 c J_{\mathbf{q}, 0}^{z}\left[\sqrt{N} M_{2}\left(\beta h_{0}\right)-\left(c-1+c S_{2}(\mathbf{q})\right) y\left(2 M_{1}\left(\beta h_{0}\right)+y\right)\right]\right) \\
& +\frac{1}{4 \beta} \sum_{\mathbf{q}}\left(\ln \left\{1-\mathrm{e}^{\beta h_{0}+4 c \sqrt{N} M_{1}\left(\beta h_{0}\right) J_{q, 0}^{-}}\right\} /\left\{1-\mathrm{e}^{\beta h_{0}}\right\}\right) .
\end{aligned}
$$

The obtained equations for magnetization of a localized spin subsystem are

$$
y\left[c^{2} N J_{0}+\sum_{\mathbf{q} \neq \mathbf{0}} J_{q} K_{2}(q ; c)+2 \beta \sum_{\mathbf{q} \in \mathbf{\Lambda}} I_{q}^{2} K_{2}(q ; c) \chi^{z z}(\mathbf{q}, 0)\right]-c^{2} N J_{0} M_{1}\left(\beta h_{0}\right)
$$




$$
\begin{aligned}
& +\frac{c J_{0}}{4}\left(\frac{1+4 c \sqrt{N} J_{q, 0}^{-} M_{2}\left(\beta h_{0}\right)}{1-\mathrm{e}^{-\beta h_{0}-4 c \sqrt{N} J_{q, 0}^{-} M_{1}\left(\beta h_{0}\right)}}-\frac{1}{1-\mathrm{e}^{-\beta h_{0}}}\right) \\
& +\frac{c}{\beta} \sum_{\mathbf{q} \in \boldsymbol{\Lambda}} \frac{J_{\mathbf{q}, \mathbf{0}}^{z}\left(\beta c \sqrt{N} J_{0} M_{3}\left(\beta h_{0}\right)-2 K_{2}(q ; c)\left[\beta c J_{0} M_{2}\left(\beta h_{0}\right) y+M_{1}\left(\beta h_{0}\right)+y\right]\right)}{1+2 c J_{\mathbf{q}, \mathbf{0}}^{z}\left[\sqrt{N} M_{2}\left(\beta h_{0}\right)-K_{2}(q ; c) y\left(2 M_{1}\left(\beta h_{0}\right)+y\right]\right.}=0,
\end{aligned}
$$

and the equation for electron spin polarization is

$$
\begin{aligned}
& \frac{c}{N} \sum_{\mathbf{q} \in \boldsymbol{\Lambda}} \frac{J_{\mathbf{q}, \mathbf{0}}^{z}\left(\sqrt{N} M_{3}\left(\beta h_{0}\right)-2 K_{2}(q ; c) M_{2}\left(\beta h_{0}\right) y\right)}{1+2 c J_{\mathbf{q}, \mathbf{0}}^{z}\left[\sqrt{N} M_{2}\left(\beta h_{0}\right)-K_{2}(q ; c) y\left(2 M_{1}\left(\beta h_{0}\right)+y\right)\right]} \\
& \quad+c\left[y-M_{1}\left(\beta h_{0}\right)\right]+\frac{1}{4 \varrho} \sum_{\mathbf{q} \in \mathbf{\Lambda}}\left(\frac{1+4 c \sqrt{N} J_{q, 0}^{-} M_{2}\left(\beta h_{0}\right)}{1-\mathrm{e}^{-\beta h_{0}-4 c \sqrt{N} J_{q, 0}^{-} M_{1}\left(\beta h_{0}\right)}}-\frac{1}{1-\mathrm{e}^{-\beta h_{0}}}\right)=0 .
\end{aligned}
$$

The equation for the chemical potential in the given approximation remains the same as in the self-consistent field approach and is given by the last equation of system (72).

\section{The self-consistent field approximation}

We shall neglect in the Hamiltonian (30) the term $\widehat{H}_{\text {int }}$ which describes the correlation spin-spin and spin-electron interactions. Then the effective Hamiltonian reads

$$
\widehat{H}_{\mathrm{eff}}=C+\widehat{H}_{0}=C-\sum_{j=1}^{N} \hat{c}_{j} \widetilde{h}_{j} S_{j}^{z}+\sum_{\mathbf{k}} \sum_{\sigma=\uparrow, \downarrow} \widetilde{\mathcal{E}}_{k, \sigma} a_{\mathbf{k}, \sigma}^{+} a_{\mathbf{k}, \sigma} .
$$

A random external magnetic field $\widetilde{h}_{j}$, renormalized spectrum of free electrons $\widetilde{\mathcal{E}}_{k, \sigma}$, and non-operator part of the Hamiltonian are given by the relations (33), (34) and (33). Such an approximation is equivalent to the approximation of the self-consistent mean field. Peculiar to this problem in our case, in contrast to the ideal crystal magnetics, is a structural disorder of the model which requires additional analysis and calculations.

Thermodynamic potential in the approximation of the mean self-consistent field for a fixed configuration of atoms of the system

$$
\Omega_{0}\left[\hat{c}^{N} \mathbf{R}^{N}\right]=-\frac{1}{\beta} \ln \operatorname{Sp}_{\mathrm{s}} \operatorname{Sp}_{\mathrm{e}} \mathrm{e}^{-\beta\left(C+\widehat{H}_{0}-\mu \widehat{N}_{\mathrm{e}}\right)}
$$

is easy to calculate and reduces to the analytical expression (52). The last term in this formula is the thermodynamic potential of a subsystem of noninteracting magnetic atoms in a random magnetic field.

Now it is necessary to average the thermodynamical potential over all possible configurations. For this purpose we shall separate a structurally dependent component of the field $\widetilde{h}_{j}$

$$
\widetilde{h}_{j}=h_{0}+\Delta_{j}
$$


where the spatially homogeneous component of the self-consistent magnetic field is

$$
h_{0}=g \mu_{\mathrm{B}} h+\frac{1}{\varrho} p I_{0}+c y J_{0}
$$

and the quantity

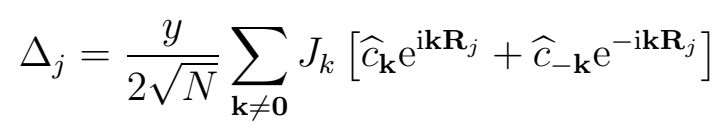

characterizes a deviation of a field at the point $\mathbf{R}_{j}$ from the average value. It is obvious that the configurational average $\overline{\Delta_{j}}=0$. We will suppose that the fluctuation $\Delta_{j}$ is small. As a criterion of smallness of fluctuations of the magnetic field acting on the spin of the atom localized at the point $\mathbf{R}_{j}$ it is convenient to use the parameter

$$
\gamma=\frac{\sqrt{\frac{1}{N} \sum_{1 \leqslant j \leqslant N} \overline{\hat{c}_{j} \Delta_{j}^{2}}}}{y c J_{0}}=v_{\mathrm{M}} \sqrt{\frac{1}{V^{2}} \sum_{\mathbf{k}} \sum_{\mathbf{q}} \widetilde{J}_{\mathbf{k}} \widetilde{J}_{\mathbf{q}} K_{3}(\mathbf{k} ; \mathbf{q} ;-\mathbf{k}-\mathbf{q})}
$$

which is the ratio of the mean-arithmetic value of a linear dispersion of the distribution to the value of the average field. Here $\widetilde{J}_{\mathbf{k}}=J_{\mathbf{k}} / J_{0}$ is the normalized coefficient of interaction, and $v_{\mathrm{M}}=V / N_{\mathrm{M}}=1 / c \rho$ is the volume per one magnetic atom. The parameter $\gamma$ will be small under condition of large concentrations of magnetic atoms and long-range spin-spin interactions. The concrete numerical estimation of parameter can be performed, having a model interaction law and an expression for the correlation function $K_{3}$.

Let us expand the expression (52) into a power series over the fluctuations $\Delta_{j}$. After cumbersome transformations, we obtain the following final expression for the configurationally averaged value of thermodynamic potential $\Omega_{0}(\beta ; c)=\overline{\Omega_{0}\left[\hat{c}^{n} \mathbf{R}^{n}\right]}$ in the approximation of self-consistent field

$$
\begin{aligned}
& \Omega_{0}(\beta ; c)=\frac{1}{2} c N S(S+1) J(0)+y p V I_{0}+\frac{1}{2} c^{2} y^{2} N J_{0}+\frac{y^{2}}{2} \sum_{\mathbf{k} \neq \mathbf{0}} J_{k} K_{2}(k) \\
& \quad-\frac{1}{\beta} \sum_{\mathbf{k}} \sum_{\sigma=\uparrow, \downarrow} \ln \left[1+\mathrm{e}^{-\beta\left(\widetilde{\mathcal{E}}_{k, \sigma}-\mu\right)}\right]-\frac{c N}{\beta} \ln \left[\frac{\sinh \left(\beta h_{0} \frac{2 S+1}{2}\right)}{\sinh \left(\frac{1}{2} \beta h_{0}\right)}\right] \\
& \quad+\sum_{n \geqslant 1} \frac{y^{n} \beta^{n-1} M_{n}\left(\beta h_{0}\right)}{N^{n-1} n !} \sum_{\mathbf{k}_{\mathbf{1}} \neq \mathbf{0}} \ldots \sum_{\mathbf{k}_{\mathbf{n}} \neq \mathbf{0}} J_{\mathbf{k}_{\mathbf{1}}} \cdots J_{\mathbf{k}_{\mathbf{n}}} K_{n+1}\left(\mathbf{k}_{\mathbf{1}} ; \ldots ; \mathbf{k}_{\mathbf{n}} ; \mathbf{k}_{\mathbf{1}}-\ldots-\mathbf{k}_{\mathbf{n}}\right) .
\end{aligned}
$$

Functions $M_{n}(x)$ satisfy the recurrent relations $M_{n}(x)=\mathrm{d} / \mathrm{d} x M_{n-1}(x), n \geqslant 1$. Here $M_{0}(x)=\ln [\sinh (0.5 x[2 S+1]) / \sinh (0.5 x)]$, the first derivative is $M_{1}(x)=$ $S B_{S}(x S)$, where $B_{S}(x)$ is the Brillouin function.

The obtained expression for thermodynamic potential of the spin-electron model in mean field approximation is a generalization of results known in the theory of magnetism to the case of an amorphous binary substitutional alloy, with making use of a special, more accurate for structurally disordered systems, method of allocation 
of self-consistent fields. Expression (69) contains contributions to the thermodynamic potential due to structural fluctuations being taken into account. We shall make the following remark about this fact. In the thermodynamic potential $\Omega_{0}(\beta ; c)$ does not contain contributions from spin fluctuations, since the mean field approximation is used. The terms which contain the sum over wave vectors are due to structural fluctuations being taken into account. Using the thermodynamic potential in the form (69) to explore the thermodynamic properties of spin-electron model will not be correct, and the drawn physical conclusions will be invalid. The fact is that the contributions of the same order to the thermodynamic potential will be given by the correlation energy $\widehat{H}_{\text {int }}$ being taken into account, which is neglected at the present stage.

\section{Magnetizations and critical temperature of spin-electron model. The self-consistent field approximation}

Let us find the equation for magnetization in the simplest approximation, when the contribution of structural fluctuations in thermodynamic potential (69) is neglected, i.e. we shall take those correlation functions $K_{n}\left(\mathbf{k}_{1} ; \ldots ; \mathbf{k}_{n}\right) \equiv 0, n \geqslant 1$. Actually, as noted above, such terms being taken into account in the approximation of the self-consistent field cannot be validated.

From the condition of a minimum of thermodynamic potential with respect to the order parameters $y$ and $p$

$$
\frac{\mathrm{d} \Omega_{0}(\beta ; c)}{\mathrm{d} y}=0, \quad \frac{\mathrm{d} \Omega_{0}(\beta ; c)}{\mathrm{d} p}=0
$$

and the equation for the chemical potential of the electron subsystem of the alloy

$$
\rho_{\mathrm{e}}=\frac{1}{V} \sum_{\mathbf{k}}\left[\frac{1}{1+\mathrm{e}^{\beta\left(\widetilde{\mathcal{E}}_{k, \uparrow}-\mu\right)}}+\frac{1}{1+\mathrm{e}^{\beta\left(\widetilde{\mathcal{E}}_{k, \downarrow}-\mu\right)}}\right],
$$

where $\rho_{\mathrm{e}}=N_{\mathrm{e}} / V$ is the density of conductivity electrons, we obtain a system of equations for $y, p$ and $\mu$ :

$$
\begin{aligned}
M & =B_{\mathrm{s}}\left(\frac{S}{T}\left[g \mu_{\mathrm{B}} h+c S M J_{0}+\frac{1}{2} P n_{\mathrm{e}} I_{0}\right]\right), \\
\frac{4}{3}\left(\frac{E_{\mathrm{F}}}{T}\right)^{3 / 2} P & =F_{\frac{1}{2}}\left(\frac{\mu+\xi}{T}\right)-F_{\frac{1}{2}}\left(\frac{\mu-\xi}{T}\right), \\
\frac{4}{3}\left(\frac{E_{\mathrm{F}}}{T}\right)^{3 / 2} & =F_{\frac{1}{2}}\left(\frac{\mu+\xi}{T}\right)+F_{\frac{1}{2}}\left(\frac{\mu-\xi}{T}\right) .
\end{aligned}
$$

The system (72) was obtained using the expression for the density of states of free electrons $\rho_{0}(E)=3 / 4 \cdot N_{\mathrm{e}} \sqrt{E} \cdot E_{\mathrm{F}}^{-3 / 2}$, where Fermi energy of electron gas is $E_{\mathrm{F}}=\left(3 \pi^{2}\right)^{2 / 3} \hbar^{2} \rho_{\mathrm{e}}^{2 / 3} / 2 m$. The function $F_{0.5}(\alpha)$ is Fermi-Dirac integral. Here 
$\xi=\mu_{\mathrm{B}} h+c I_{0} S M / 2$, and the reduced (normalized per the value of a localized spin) magnetization of an atom is $M=y / S$. In the equations (72) the transition to the values convenient in calculations is made: $P=2 p V / N_{\mathrm{e}}$ is the electron spin polarization per one electron, which can take values $\widetilde{m} \in[-1 ; 1]$ (in Bohr magnetons). The quantity $n_{\mathrm{e}}=N_{\mathrm{e}} / N$ is the number of electrons per one atom. Numerical solution of system (72) enables one to investigate the dependences of magnetizations $M$ and $P$ on temperature at different values of parameters $J_{0}, I_{0}, n_{\mathrm{e}}, S, E_{\mathrm{F}}$.

Let us examine the value of electron spin polarization at zero temperature. In this case we shall not consider the first equation of the system, replacing everywhere $M=1$, i.e. by the maximal value of the relative magnetization of atoms. We shall suppose also that the condition $c S\left|I_{0}\right| / 2 E_{\mathrm{F}}<1$ is satisfied. In general, practically for all ferromagnetic metals and wide-zone semiconductors a stronger condition $c S\left|I_{0}\right| / 2 E_{\mathrm{F}} \ll 1$ is obeyed. That is so due to the fact that the energy $E_{\mathrm{F}}$ at actual densities of electrons is large, order of $E_{\mathrm{F}} \sim 10^{4} \mathrm{~K}$, and the parameter of spin-electron interaction is $I_{0} \sim 10^{2} \mathrm{~K}$. Under such conditions it is possible to use the asymptotics of the Fermi-Dirac function $F_{n}(\alpha)=\alpha^{n+1} /(n+1)$ for large values of its argument.

From the equation for chemical potential we obtain an approximate formula, which describes a shift of chemical potential at zero temperature $\mu_{0}=\mu(T=0)$ due to spin-electron interaction

$$
\mu_{0}=E_{\mathrm{F}}\left[1+\frac{2}{3} \frac{2-\left(1+\frac{c I_{0} S}{2 E_{\mathrm{F}}}\right)^{\frac{3}{2}}-\left(1-\frac{c I_{0} S}{2 E_{\mathrm{F}}}\right)^{\frac{3}{2}}}{\sqrt{1+\frac{c I_{0} S}{2 E_{\mathrm{F}}}}+\sqrt{1-\frac{c I_{0} S}{2 E_{\mathrm{F}}}}}\right] .
$$

When $c S\left|I_{0}\right| / 2 E_{\mathrm{F}} \ll 1$ it simplifies to

$$
\mu_{0}=E_{\mathrm{F}}\left[1-\frac{c^{2} I_{0}^{2} S^{2}}{8 E_{\mathrm{F}}^{2}}+\cdots\right]
$$

Hence, a shift of Fermi energy under the effect of spin-electron interaction in ferromagnetic metals is small, and this effect can be neglected.

From the second equation of the system (72) we obtain a simple expression for electron magnetization at zero temperature $P_{0} \equiv P(T=0)$

$$
P_{0}=\frac{1}{2}\left[\left(1+\frac{c S I_{0}}{2 E_{\mathrm{F}}}\right)^{3 / 2}-\left(1-\frac{c S I_{0}}{2 E_{\mathrm{F}}}\right)^{3 / 2}\right] \text {. }
$$

Formally this expression makes sense at $0 \leqslant \frac{c S I_{0}}{2 E_{\mathrm{F}}} \leqslant 1$. However at the upper limit of this interval the electron magnetization gets nonphysical values $P_{0}=\sqrt{2}$. It indicates that the equation (75) is applicable only at $\frac{c S I_{0}}{2 E_{\mathrm{F}}} \ll 1$.

If one takes into account the renormalization of the Fermi energy due to spinelectron interaction then

$$
P_{0}=\frac{1}{2}\left[\left(1+\frac{c S I_{0}}{2 E_{\mathrm{F}}}-\frac{c^{2} S^{2} I_{0}^{2}}{8 E_{\mathrm{F}}^{2}}\right)^{3 / 2}-\left(1-\frac{c S I_{0}}{2 E_{\mathrm{F}}}-\frac{c^{2} S^{2} I_{0}^{2}}{8 E_{\mathrm{F}}^{2}}\right)^{3 / 2}\right] .
$$


This formula is applicable for $0 \leqslant c S I_{0} / 2 E_{\mathrm{F}} \leqslant \sqrt{2}-1$, when at the upper limit the physically allowable value of magnetization $P_{0} \simeq 4 / 5$ is obtained. However at values $c S I_{0} / 2 E_{\mathrm{F}}$ close to unity, results of calculations can be doubtful due to the approximations made in obtaining the equation (76).

We can see from the equation (76) that electron magnetization $\widetilde{m}_{0}$ is always smaller than its maximal possible value: $\left|P_{0}\right|=1$. This effect is of a quantum origin and could be explained by the Pauli blocking rule. For the first time such an effect has been found in the Stoner model in which, using the concept of a molecular field and Fermi statistics, the problems of occurrence of ferromagnetic ordering of conductivity electrons in ferromagnetic metals were studied.

If $I_{0}>0$ (ferromagnetic spin-electron interaction), then $P$ in (76) will be positive, otherwise if $I_{0}<0$ (antiferromagnetic spin-electron interaction) the values of electron spin polarization $P$ are negative. In the first case the spin and electron magnetizations will be codirected and in the second - opposite directed, i.e. they will partially compensate each other.
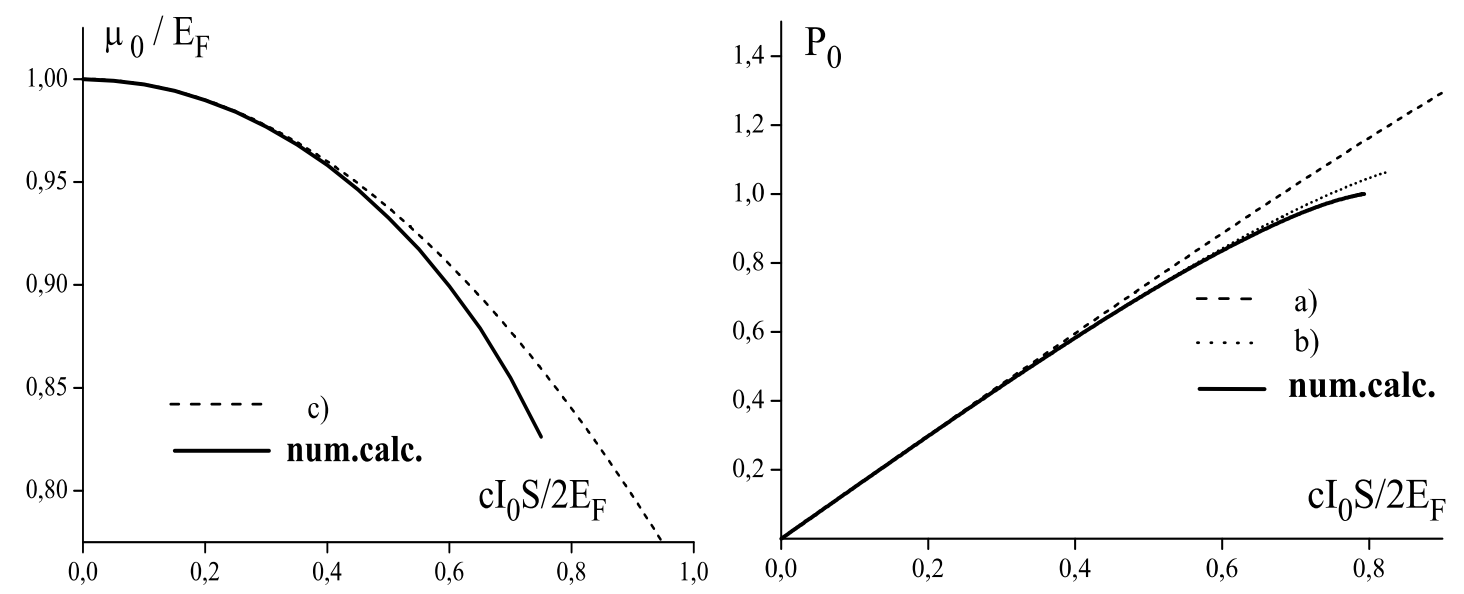

Figure 1. Chemical potential and electron magnetization at $T=0:$ a) - equation (74), b) - equation (75), c) - equation (76).

In figure 1 the dependence of chemical potential $\mu_{0}$ at $T=0$ calculated over the equation (74) on the parameter $c S I_{0} / 2 E_{\mathrm{F}}$ is shown, as well as the results of the exact numerical calculations for the system of the equations (72). The right part of figure 1 contains the dependence of the electron spin polarization on the same parameter calculated over the approximate formulas (75), (76) as well as the results of exact numerical calculations. At small values of $c S I_{0} / 2 E_{\mathrm{F}}$ the linear behaviour of electron spin polarization is observed. It is seen that the approximate extrapolation formulas give quantitatively good values in the region $0 \leqslant c S I_{0} / 2 E_{\mathrm{F}}<0.5$.

In figure 2 the dependence of electron spin polarization and magnetization of a subsystem of localized spins from temperature is shown at different values of the model parameters. Results are obtained by numerical solving of the system of the equations (72). The temperature is given in units of $T_{\mathrm{c}}^{0}=c S(S+1) J_{0} / 3$ - of Curie temperature of Heisenberg magnet in the molecular field approximation. 

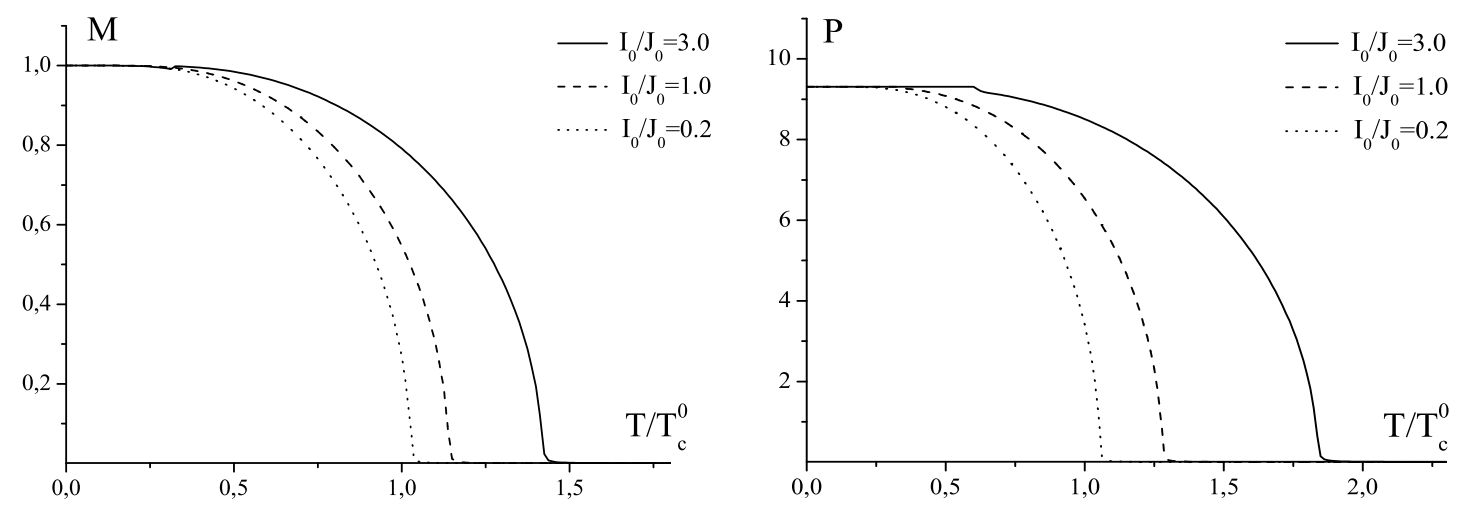

Figure 2. Electron spin polarization and magnetization of a subsystem localized spins at $T=0$.

An important characteristic of magnetic systems is the temperatures of phase transitions. We shall find the temperature of transition from the disordered paramagnetic to the ordered ferromagnetic phase (the so-called Curie temperature $T_{\mathrm{c}}$ ). For this purpose we shall put the external magnetic field equal to zero. At $T \rightarrow T_{\mathrm{c}}$ we have $\xi=c I_{0} S M / 2 \rightarrow 0$. Chemical potential of the electron subsystem is an analytical function of temperature. Let us introduce the notation $\mu_{\mathrm{c}}=\mu\left(T=T_{\mathrm{c}}\right)$. We shall expand the right sides of $(72)$ in the vicinity of temperature $T_{\mathrm{c}}$ into the ascending power series up to the linear in $M$ and $m$ terms:

$$
\begin{aligned}
M & =\frac{S(S+1)}{3 T_{\mathrm{c}}}\left(c J_{0} M+\frac{1}{2} P n_{\mathrm{e}} I_{0}\right), \\
\frac{4}{3}\left(\frac{E_{\mathrm{F}}}{T_{\mathrm{c}}}\right)^{3 / 2} P & =2 F_{\frac{1}{2}}^{\prime}\left(\frac{\mu_{\mathrm{c}}}{T_{\mathrm{c}}}\right) \frac{c S I_{0} M}{2 T_{\mathrm{c}}}, \\
\frac{4}{3}\left(\frac{E_{\mathrm{F}}}{T_{\mathrm{c}}}\right)^{3 / 2} & =2 F_{\frac{1}{2}}\left(\frac{\mu_{\mathrm{c}}}{T_{\mathrm{c}}}\right) .
\end{aligned}
$$

Here we denoted $F^{\prime}{ }_{n}(x)=\mathrm{d} F_{n}(x) / \mathrm{d} x$. Two first equations form a system of equations, uniform with respect to $M$ and $P$. It has non-trivial solutions if the main determinant of the system is equal to zero. From this condition we obtain an equation for the Curie temperature:

$$
1-\frac{c S(S+1) J_{0}}{3 T_{\mathrm{c}}}=\frac{c S(S+1)}{8 T_{\mathrm{c}}^{2}} n_{\mathrm{e}} I_{0}^{2}\left(\frac{T_{\mathrm{c}}}{E_{\mathrm{F}}}\right)^{3 / 2} F_{\frac{1}{2}}^{\prime}\left(\frac{\mu_{\mathrm{c}}}{T_{\mathrm{c}}}\right) .
$$

The equation (78) is transcendent. We can make estimations for some limiting cases. So if concentration of electrons $n_{\mathrm{e}}$ or parameter $I_{0}$ are equal to zero, the solution of equation (78) will be the critical temperature for the ideal Heisenberg model. If $J_{0}$ is zero (classical $s$ - $d$ model of Shubin-Vonsovsky), we obtain the following 
equation

$$
1=\frac{c S(S+1)}{8 T_{\mathrm{c}}^{2}} I_{0}^{2} \frac{1}{\varrho}\left(\frac{T_{\mathrm{c}}}{E_{\mathrm{F}}}\right)^{3 / 2} F_{\frac{1}{2}}^{\prime}\left(\frac{\mu}{T_{\mathrm{c}}}\right) .
$$

Using the asymptotical expression for the Fermi-Dirac function we find

$$
T_{\mathrm{c}}=\frac{c S(S+1)}{8} \frac{I_{0}^{2}}{E_{\mathrm{F}}} \frac{1}{\varrho} .
$$

Similarly, we shall obtain an estimation for the full equation (78)

$$
\frac{T_{\mathrm{c}}}{T_{\mathrm{c}}^{o}}=1+\frac{9}{8} \frac{1}{c S(S+1)}\left(\frac{I_{0}}{J_{0}}\right)^{2} \frac{1}{\varrho} \frac{T_{\mathrm{c}}^{o}}{E_{\mathrm{F}}} .
$$

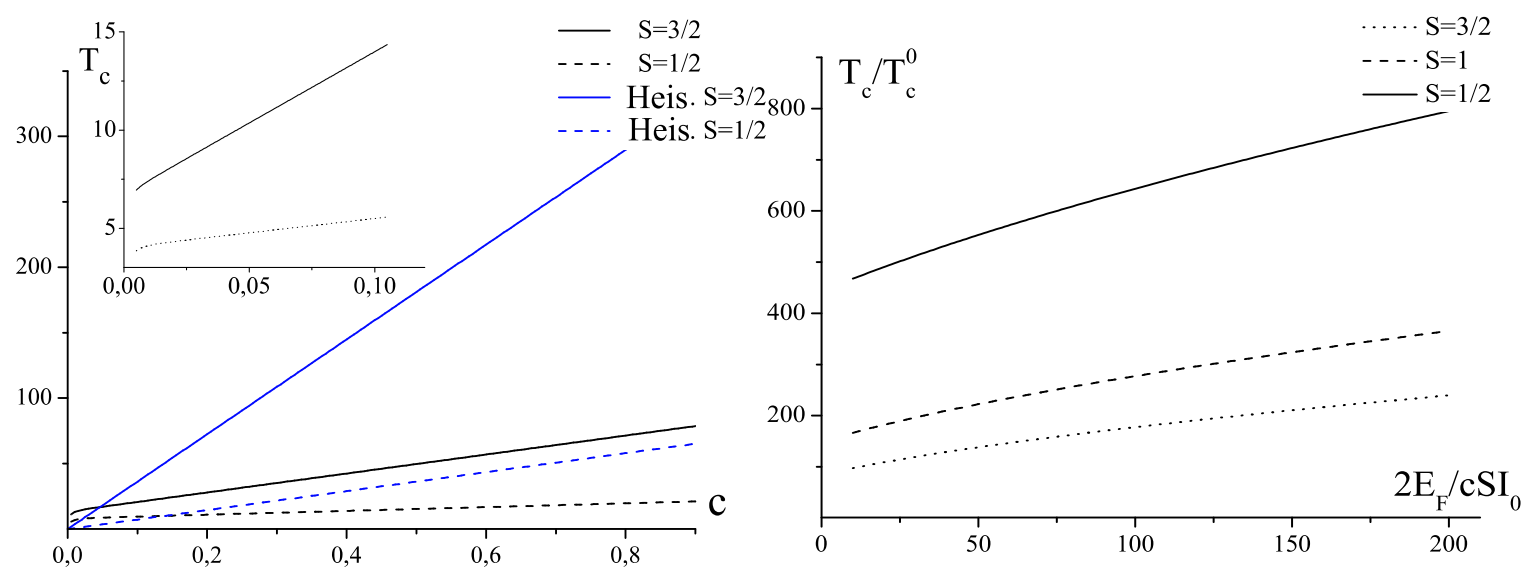

Figure 3. Dependence of Curie temperature on concentration of magnetic impurities and ratio of Fermi energy to the spin-electron interaction integral.

In figure 3 the results of numerical calculations of critical temperature are presented at some values of the model parameters. Calculations are performed for the case of metal alloys, when parameter $c S\left|I_{0}\right| / 2 E_{\mathrm{F}} \ll 1$.

\section{Nondirect spin-spin interaction in ferromagnetic phase}

From the equation (71) one can draw an important conclusion: in the presence of magnetic ordering in the system the indirect pair spin-spin interaction is anisotropic, with the preferential direction along the axis of quantization. In the paramagnetic phase such an interaction becomes isotropic, which is typical of the RKKY interaction.

In general, to obtain an interaction of the RKKY type from expression (71), we put the self-consistent field equal to zero (the absence of external magnetic field and a paramagnetic phase), replace the Fourier components $I_{k}$ with the constant $I$ for all values of wave vectors (contact spin-electron interaction), put the frequency $\omega$ 
equal to zero in a susceptibility $\chi^{\alpha_{1}, \alpha_{2}}(\mathrm{k} ; \omega)$, and pass to the limit of absolute zero temperature. Then the Fourier components of the effective spin-spin interaction are

$$
J_{\text {eff }}(k)=\lim _{T \rightarrow 0} \frac{\beta I^{2}}{2} \chi(\mathbf{k}, 0)=\frac{I^{2} m}{16 \varrho \hbar^{2} \pi^{2}} \sum_{\sigma=\uparrow, \downarrow} q_{v \sigma}\left[\frac{4 q_{v \sigma}^{2}-k^{2}}{4 k q_{v \sigma}} \ln \left|\frac{k+2 q_{v \sigma}}{k-2 q_{v \sigma}}\right|+1\right]
$$

here

$$
q_{v \sigma}=\sqrt{2 m\left\{\mu-\varkappa_{\sigma}\left(\mu_{\mathrm{B}} h+y c I_{0}\right)-w_{0}\right\}} / \hbar
$$
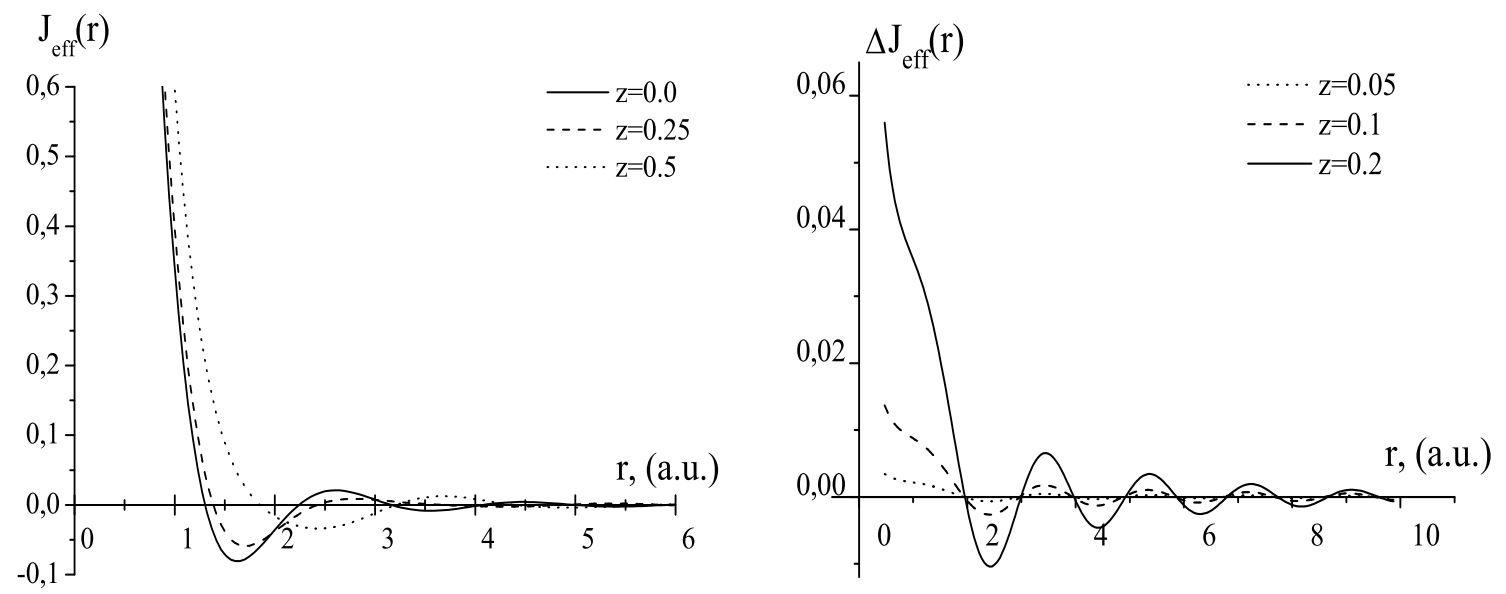

Figure 4. The typical behaviour of RKKY type interaction (figure at left side) and normalized by interaction without spin-electron effect (figure at right side)

In the coordinate representation

$$
J_{\text {eff }}(r)=\frac{I^{2} k_{F}^{2}}{16 \pi \varrho E_{\mathrm{F}}} \sum_{\sigma=\uparrow, \downarrow} q_{v \sigma}^{4} \frac{\sin \left(2 q_{v \sigma} r\right)-2 q_{v \sigma} r \cos \left(2 q_{v \sigma} r\right)}{\left(q_{v \sigma} r\right)^{4}}
$$

we receive some complication of the known form of nondirect spin-spin interaction.

Here $z=c y I_{0} / 2 E_{\mathrm{F}}, J_{\mathrm{eff}}(r)$ means the energy in units normalized by magnitude

$$
\frac{I^{2} k_{F}^{2}}{16 \pi \varrho E_{\mathrm{F}}} q_{v \sigma}^{4} \text { and } \text { the } \Delta J_{\mathrm{eff}}(r)=J_{\mathrm{eff}}^{0}(r)-\frac{\left(J_{\mathrm{eff}}^{\uparrow}(r)+J_{\mathrm{eff}}^{\downarrow}(r)\right)}{2}
$$

normalized of the same magnitude too. The parameter $z=0$ in case of the absence of an exterior magnetic field.

In figure 4 one can see that with injection in metal of some concentrations of localized atom moments the spins of conduction electrons create oscillating polarization near it. The oscillations of spin density are similar to the Friedel oscillation of charge density. 


\section{Conclusions}

From expression (81) one can see that the presence of exchange spin-electron interaction results in the increase of critical temperature. It could be explained by increasing the effective spin-spin interaction due to spin-electron interaction. The contribution of the electron subsystem to magnetization and the value of critical temperature enhance with the increase of electron concentration $n_{\mathrm{e}}$ and the parameter of exchange spin-electron interaction $I_{0}$.

The obtained above results for magnetizations of the subsystems of localized spins and of conductivity electrons, as well as the critical temperature of phase transition in the self-consistent field approximation qualitatively correctly display the known experimental data of a certain class of magnetic alloys.

However, for a quantitative description of properties of magnetic alloys, in particular, in the absence of small parameters and at small concentrations (densities) of magnetoactive atoms, it is necessary to take into account the structural and thermodynamical spin fluctuations, as done in section 4. Without the high-order fluctuations being consistently taken into account it is impossible to explain some observable properties even qualitatively. It concerns, for example, the features of a spectrum of elementary excitations in the short-wave regions $(k a \sim 1)$, the percolation limit of the critical temperature, features of the system behaviour in the vicinity of a critical point and so forth.

\section{References}

1. Vonsovsky S.V. Magnetizm. Moskva, Nauka, 1971 (in Russian).

2. Nagajev E.L. Fizika magnitnyh poluprovodnikov. Nauka, Moskva, 1979 (in Russian).

3. Metfessel Z., Mattis D. Magnitnyje poluprovodniki. Mir, Moskva, 1972 (in Russian).

4. Izumov Yu.A., Katsnelson M.I., Skriabin Yu.N. Magnetizm kollektivizirovannyh elektronov. Fizmatlit, Moskva, 1994 (in Russian).

5. Stasyuk I., Shvajka A., Tabunshchyk K., Condens. Matter Phys., 1999, 2, 109-132.

6. Stasyuk I.V. Pseudospin-electron model for strongly correlated electron system (Thermodynamics and dynamamics). - In: AIP Conference Proceedings. Highlights in condensed matter physics, 2003, 695, 281-290.

7. Izumov Yu.A., Kassan-Ogly F.A., Skriabin Yu.N. Polevyje metody v teorii ferromagnetizma. Nauka, Moskva, 1974 (in Russian).

8. Letfulov B.M., FTT, 1976, 18, 645.

9. Maćkowiak J., Wiśniewski M., Physica A, 1997, 242, 482.

10. Công B.T., Phys. Stat. Sol. (b), 1986, 134, 569.

11. Vakarchuk I.A., Rudavskii Yu.K., TMF, 1984, 58, 445.

12. Vakarchuk I.A., Rudavskii Yu.K., Ponedilok G.V, Phys. Stat. Sol. (b), 1985, 128, 231.

13. Ponedilok G.V., Savenko V.P., Phys. Stat. Sol. (b), 1994, 184, 433.

14. Dovgopol S.P., Kroxin A.L., Mirzojev A.A., TMF, 1979, 41, 378.

15. Brovman E.G., Kagan Yu.M., UFN, 1974, 122, 3, 369.

16. Vavruch M.V., Krochmalskii T.Ye., Ukrainian Journal of Physics, 1987, 32, 648.

17. Ianarella L., Guimaraes A.P., Silva X.A., Phys. Stat. Sol. (b), 1982, 114, 255.

18. Vakarchuk I.O., Tkachuk V.M., Kuliy T.V., Phys. Stat. Sol. (b), 1998, 208, 167. 


\section{Термодинаміка структурно невпорядкованої $s-d$ моделі}

\section{Ю.Рудавський, Г.Понеділок, Л.Дорош}

Національний університет “Львівська політехніка", вул. С.Бандери 12, Львів 79013, Україна

Отримано 5 травня 2004 р., в остаточному вигляді 22 жовтня 2004 р.

Спін-електронна обмінна модель узагальнюється і застосовується для опису магнітних станів аморфних сплавів з рідиноподібним типом структурної невпорядкованості. Розглянута схема послідовного врахування вкладу структурних флуктуацій у термодинамічні функції та спостережувані величини. За теорією збурень побудовано функціонал термодинамічного потенціалу у формі функціонального степеневого ряду. У наближенні хаотичних фаз (RPA) розраховано великий термодинамічний потенціал моделі. Записані співвідношення самоузгодження, з яких знаходяться рівняння для намагніченостей та критичної температури переходу "парамагнетикферомагнетик".

Ключові слова: $s-d$-модель, феромагнітні сплави, вільна енергія, намагніченість, температура Кюрі, функціональні інтеграли

PACS: 72.15.C, 73.20.H, 75.10, 75.30.E, 82.65.Y 